\title{
PARABOLIC AND ELLIPTIC SYSTEMS WITH VMO COEFFICIENTS*
}

\author{
HONGJIE DONG ${ }^{\dagger}$ AND DOYOON KIM ${ }^{\ddagger}$
}

\begin{abstract}
We consider second order parabolic and elliptic systems with leading coefficients having the property of vanishing mean oscillation (VMO) in the spatial variables. An $L_{q}-L_{p}$ theory is established for systems both in divergence and non-divergence form.
\end{abstract}

Key words. Second order parabolic and elliptic systems, Vanishing mean oscillation (VMO) coefficients, $L_{q}-L_{p}$ estimates, Sobolev spaces.

AMS subject classifications. 35K40, 35J47, 35R05, 35D35

1. Introduction. The $L_{p}$ theory of second order parabolic and elliptic equations has been studied extensively by many authors for more than fifty years. It is of particular interest not only because of its various important applications in nonlinear equations, but also due to its subtle links with the theory of stochastic processes. For scalar equations, the solvability theory in $L_{p}$ spaces has been well established; see, for example, [7], [8], [11], [25], [3], [18]-[23], [4] and references therein.

For elliptic systems with discontinuous coefficients, there are also quite a few results in the literature. Local $L_{p}$ and Hölder estimates of elliptic systems in nondivergence form with VMO coefficients were obtained in [29]. We also would like to bring attention to an interesting paper [5], in which the authors obtain the $W_{p}^{1}$ solvability of a conormal derivative problem of divergence elliptic systems with VMO coefficients without lower order terms in Reifenberg flat domains. For other results about elliptic systems with discontinuous coefficients, see also [6], [9], [2], [16], [12], [31], [24], [28] and references therein.

In contrary, until quite recently, there are not as many results of $L_{p}$ theory for parabolic systems with discontinuous coefficients. On the other hand, quite naturally, many evolutionary equations arising from physical and economical problems are coupled systems instead of scalar equations, such as the Navier-Stokes equations.

In this paper we consider two types of parabolic operators

$$
\begin{aligned}
& P \boldsymbol{u}=-\boldsymbol{u}_{t}+A^{\alpha \beta} D_{\alpha \beta} \boldsymbol{u}+B^{\alpha} D_{\alpha} \boldsymbol{u}+C \boldsymbol{u}, \\
& \mathcal{P} \boldsymbol{u}=-\boldsymbol{u}_{t}+D_{\alpha}\left(A^{\alpha \beta} D_{\beta} \boldsymbol{u}+\hat{B}^{\alpha} \boldsymbol{u}\right)+B^{\alpha} D_{\alpha} \boldsymbol{u}+C \boldsymbol{u}
\end{aligned}
$$

acting on (column) vector-valued function $\boldsymbol{u}=\left(u^{1}, \cdots, u^{m}\right)^{T}$ given on

$$
\mathbb{R}^{d+1}=\left\{(t, x): t \in \mathbb{R}, x=\left(x_{1}, \ldots, x_{d}\right) \in \mathbb{R}^{d}\right\} .
$$

Here we use the notation

$$
D_{\alpha} \boldsymbol{u}=\boldsymbol{u}_{x_{\alpha}}, \quad D_{\alpha \beta} \boldsymbol{u}=\boldsymbol{u}_{x_{\alpha} x_{\beta}} \quad(\alpha, \beta=1, \ldots, d)
$$

\footnotetext{
*Received July 6, 2009; accepted for publication October 30, 2009.

${ }^{\dagger}$ Division of Applied Mathematics, Brown University, 182 George Street, Providence, RI 02912, USA (Hongjie_Dong@brown.edu). H. Dong was partially supported by a start-up funding from the Division of Applied Mathematics of Brown University and NSF grant number DMS 0800129.

${ }^{\ddagger}$ Department of Mathematics, University of Southern California, 3620 S. Vermont Avenue, KAP 108, Los Angeles, CA 90089, USA (doyoonki@usc.edu).
} 
and the usual summation convention over repeated indices is assumed. The coefficients $A^{\alpha \beta}, B^{\alpha}, \hat{B}^{\alpha}$, and $C$ are $m \times m$ matrix-valued functions given on $\mathbb{R}^{d+1}$; i.e., $A^{\alpha \beta}=$ $\left[A_{i j}^{\alpha \beta}(t, x)\right]_{m \times m}$, etc. When the coefficients $A^{\alpha \beta}, B^{\alpha}, \hat{B}^{\alpha}$, and $C^{\alpha}$ are independent of $t$, we also define and consider elliptic operators in divergence and non-divergence form

$$
\begin{aligned}
& L \boldsymbol{u}=A^{\alpha \beta} D_{\alpha \beta} \boldsymbol{u}+B^{\alpha} D_{\alpha} \boldsymbol{u}+C \boldsymbol{u}, \\
& \mathcal{L} \boldsymbol{u}=D_{\alpha}\left(A^{\alpha \beta} D_{\beta} \boldsymbol{u}+\hat{B}^{\alpha} \boldsymbol{u}\right)+B^{\alpha} D_{\alpha} \boldsymbol{u}+C \boldsymbol{u}
\end{aligned}
$$

acting on vector valued function $\boldsymbol{u}=\left(u^{1}, \cdots, u^{m}\right)^{T}$ given on $\mathbb{R}^{d}$.

We note that a similar problem is treated in a recent paper [17], where the authors considered higher order parabolic systems in non-divergence form. Under the assumption that leading coefficients are bounded, time-independent, and VMO in the spatial variables, the solvability is established in $L_{q}\left(L_{p}\right)$ spaces with $A_{p}$ Muckenhoupt weights, by using estimates of integral operators of Calderon-Zygmund type and related commutators with BMO functions. We recall that VMO consists of BMO functions whose mean oscillations on balls vanish uniformly as the radii of the balls shrink to zero. With continuous leading coefficients, similar results can be found in an earlier paper [10].

Another set of papers concerning the $L_{p}$ solvability of parabolic systems with discontinuous coefficients are [26] and [27], where the authors established the interior regularity of solutions to higher order parabolic systems in $L_{p}$ spaces and SobolevMorrey spaces, when the coefficients are VMO in both spatial and time variables. With continuous coefficients, very general mixed problems of parabolic systems in cylindrical and non-cylindrical regions were studied before in [30]. We also cite [1] for an interesting approach of gradient $L_{p}$ estimates for a class of degenerate/singular parabolic systems.

In this paper, we consider parabolic and elliptic operators in both divergence and non-divergence form. In comparison to [26], [27] and [17], the advantage of our results is that the leading coefficients are allowed to be merely measurable in the time variable and VMO in the spatial variables. This is the same class of coefficients used in [22] and [23], and is denoted as $\mathrm{VMO}_{x}$ (see the definition in Section 3). Moreover, we assume that the leading coefficients satisfy the Legendre-Hadamard condition (see Assumption 2.1, and Remark 2.2 for a comparison of different ellipticity conditions).

Under these assumptions, we establish the $L_{q}\left(L_{p}\right)$ solvability of both divergence and non-divergence form parabolic systems (cf. Theorem 2.4 and 2.5) extending the corresponding results for scalar equations in [23]. As a corollary, we also obtain the $L_{p}$ solvability for divergence and non-divergence form elliptic systems (see Theorem 2.6 and 2.7). Comparing to the result in [5], we not only treat parabolic system but also allow lower order terms, although, as a first step, in this paper we consider only second order systems in the whole space. In a forthcoming paper [14], we will extend our results to higher order parabolic (or elliptic) systems, as well as systems on a half space and on a domain.

Our approach is based on a method from [22] and [23]. Unlike the arguments in [7]-[9], [17], [29], [26]-[28] and [31], which are based on the Calderon-Zygmund theorem for certain estimates of singular integrals and the Coifman-Rochberg-Weiss commutator theorem, our proofs rely on, as in [22], [23], pointwise estimates of sharp functions of spatial derivatives of solutions. * We note that this flexible method is also

* See also [25] for a straightforward treatment of nondivergence form elliptic and parabolic equations in the Morrey space without using any singular integrals. 
applicable to equations or systems with partially VMO coefficients (see, for instance, [19], [20] and [13]). It seems unlikely that the method of singular integrals can be applied to these problems.

Roughly speaking, there are three steps in the proofs of our main theorems. In the first step, we consider systems with simple coefficients. By simple coefficients we mean coefficients depending only on the time variable. Using the Fourier transform method, we prove the $L_{2}$ solvability, from which we establish an estimate for sharp functions of derivatives of solutions to systems. To this end, we only need the Legendre-Hadamard condition. Applying the Fefferman-Stein theorem on sharp functions and the HardyLittlewood maximal function theorem, we get the solvability of systems with the simple coefficients in $L_{p}$ spaces for $p>2$, and then in $L_{p}$ spaces for $p<2$ via a duality argument. Then we get another sharp function estimate from this solvability result. In the second step, we employ the sharp function estimate obtained in the previous step and a perturbation argument to obtain the $L_{p}$ solvability of systems with leading coefficients in $\mathrm{VMO}_{x}$. Finally in the third step, adapting an approach suggested in [21], we arrive at the $L_{q}\left(L_{p}\right)$ solvability for $q \geq p$ in the non-divergence form and without this restriction in the divergence form.

To the best of our knowledge, the question of the $L_{q}\left(L_{p}\right)$ solvability in the nondivergence form for $q<p$ with $\mathrm{VMO}_{x}$ coefficients is still open even for scalar equations. We remark that such restriction is not imposed in [17]. However, our results are not covered by those in [17] because the coefficients are assumed to be timeindependent in [17].

The paper is organized as follows. The main results, Theorem 2.4, 2.5, 2.6, and 2.7 are stated in the next section. First we consider systems in non-divergence form. Section 3 is devoted to some preliminary estimates including Theorem 3.1, which may be considered as a preliminary version of Theorem 2.4. Theorem 3.1 is about the solvability of parabolic systems with simple coefficients and is proved in Section 4. Next, Theorem 5.1 is proved in Section 5 as another special case of Theorem 2.4 when $p=q$ (see also Remark 5.2). The main tool of the proof is the aforementioned pointwise estimates of sharp functions of second derivatives of solutions (Lemma 5.3). With these preparations, we are able to prove Theorem 2.4 in Section 6. In the remaining part of the paper, we turn to systems in divergence form. In Section 7, we state and prove Theorem 7.1, which is a special case of Theorem 2.5 when $p=q$, and is a counterpart of Theorem 5.1. Then Theorem 2.6 is proved in Section 8. Finally, the results of $L_{p}$ theory of elliptic systems, i.e., Theorem 2.6 and 2.7, are derived in Section 9 by using Theorem 2.4 and 2.5 .

We finish the section by introducing some notation. Throughout the paper, we always assume that $1<p, q<\infty$ unless explicitly specified otherwise. By $N(d, p, \ldots)$ we mean that $N$ is a constant depending only on the prescribed quantities $d, p, \ldots$. For a (matrix-valued) function $F(t, x)$ in $\mathbb{R}^{d+1}$, we set

$$
(F)_{\mathcal{D}}=\frac{1}{|\mathcal{D}|} \int_{\mathcal{D}} F(t, x) d x d t=f_{\mathcal{D}} F(t, x) d x d t
$$

where $\mathcal{D}$ is an open subset in $\mathbb{R}^{d+1}$ and $|\mathcal{D}|$ is the $d+1$-dimensional Lebesgue measure of $\mathcal{D}$. For $-\infty \leq S<T \leq \infty$, we set

$$
L_{q, p}\left((S, T) \times \mathbb{R}^{d}\right)=L_{q}\left((S, T), L_{p}\left(\mathbb{R}^{d}\right)\right),
$$




$$
\begin{aligned}
& \text { i.e., } F(t, x) \in L_{q, p}\left((S, T) \times \mathbb{R}^{d}\right) \text { if } \\
& \qquad \begin{array}{c}
\|F\|_{L_{q, p}\left((S, T) \times \mathbb{R}^{d}\right)}=\left(\int_{S}^{T}\left(\int_{\mathbb{R}^{d}}|F(t, x)|^{p} d x\right)^{q / p} d t\right)^{1 / q}<\infty \\
W_{q, p}^{1,2}\left((S, T) \times \mathbb{R}^{d}\right)=\left\{u: u, u_{t}, D u, D^{2} u \in L_{q, p}\left((S, T) \times \mathbb{R}^{d}\right)\right\}, \\
W_{p}^{1,2}\left((S, T) \times \mathbb{R}^{d}\right)=W_{p, p}^{1,2}\left((S, T) \times \mathbb{R}^{d}\right), \\
\mathcal{H}_{q, p}^{1}\left((S, T) \times \mathbb{R}^{d}\right)=(1-\Delta)^{1 / 2} W_{q, p}^{1,2}\left((S, T) \times \mathbb{R}^{d}\right), \\
\mathcal{H}_{p}^{1}\left((S, T) \times \mathbb{R}^{d}\right)=\mathcal{H}_{p, p}^{1}\left((S, T) \times \mathbb{R}^{d}\right), \\
\mathbb{H}_{q, p}^{-1}\left((S, T) \times \mathbb{R}^{d}\right)=(1-\Delta)^{1 / 2} L_{q, p}\left((S, T) \times \mathbb{R}^{d}\right), \\
\mathbb{H}_{p}^{-1}\left((S, T) \times \mathbb{R}^{d}\right)=\mathbb{H}_{p, p}^{-1}\left((S, T) \times \mathbb{R}^{d}\right) .
\end{array}
\end{aligned}
$$

For any $T \in(-\infty, \infty]$, we denote

$$
\mathbb{R}_{T}=(-\infty, T), \quad \mathbb{R}_{T}^{d+1}=\mathbb{R}_{T} \times \mathbb{R}^{d}
$$

\section{Main results.}

Assumption 2.1. The matrices $A^{\alpha \beta}, B^{\alpha}, \hat{B}^{\alpha}$ and $C$ satisfy

$$
\left|A^{\alpha \beta}(t, x)\right| \leq K, \quad\left|B^{\alpha}(t, x)\right| \leq K, \quad\left|\hat{B}^{\alpha}(t, x)\right| \leq K, \quad|C(t, x)| \leq K,
$$

and the Legendre-Hadamard condition

$$
A_{i j}^{\alpha \beta}(t, x) \xi_{\alpha} \xi_{\beta} \vartheta^{i} \vartheta^{j} \geq \delta|\xi|^{2}|\vartheta|^{2}
$$

for all $(t, x) \in \mathbb{R}^{d+1}, \xi \in \mathbb{R}^{d}, \vartheta \in \mathbb{R}^{m}$, for some constants $0<\delta<1$ and $K>0$.

REMARK 2.2. The Legendre-Hadamard condition is weaker and more natural than the strong ellipticity condition (also called the Legendre condition), which was used, for example, in [2], [31], [12], [24] and [5] mainly for the purpose of the $L_{2}$ estimate. See [16] for a discussion of these two conditions. However, it is still stronger than Petrovskii's condition used in [15], [9] and [26]-[29]. An interesting question is whether the results of Theorem 2.4 and 2.5 can be extended to operators satisfying the latter condition. The main difficulty in this case is to prove Theorem 3.1 when $p=2$. However, this is not an issue for elliptic operators or parabolic operators with coefficients VMO in both $x$ and $t$.

Another assumption on $A=\left[A_{i j}^{\alpha \beta}\right]$ is that they are in the class of $\mathrm{VMO}_{x}$, that is, $A_{i j}^{\alpha \beta}(t, x)$ are measurable in $t \in \mathbb{R}$ and VMO in $x \in \mathbb{R}^{d}$. A precise description of this assumption is given below using the following notation. Let

$$
B_{r}(x)=\left\{y \in \mathbb{R}^{d}:|x-y|<r\right\}, \quad Q_{r}(t, x)=\left(t-r^{2}, t\right) \times B_{r}(x) .
$$


Set $B_{r}=B_{r}(0)$ and $\left|B_{r}\right|$ to be the $d$-dimensional volume of $B_{r}$. Also set

$$
\operatorname{osc}_{x}\left(A_{i j}^{\alpha \beta}, Q_{r}(t, x)\right)=r^{-2}\left|B_{r}\right|^{-2} \int_{t-r^{2}}^{t} \int_{y, z \in B_{r}(x)}\left|A_{i j}^{\alpha \beta}(s, y)-A_{i j}^{\alpha \beta}(s, z)\right| d y d z d s
$$

and

$$
A_{R}^{\#}=\sup _{(t, x) \in \mathbb{R}^{d+1}} \sup _{r \leq R} \sum_{\alpha, \beta=1}^{d} \sum_{i, j=1}^{m} \operatorname{osc}_{x}\left(A_{i j}^{\alpha \beta}, Q_{r}(t, x)\right) .
$$

In case $A$ is independent of $t$, naturally we set

$$
\begin{gathered}
\operatorname{osc}\left(A_{i j}^{\alpha \beta}, B_{r}(x)\right)=\left|B_{r}\right|^{-2} \int_{y, z \in B_{r}(x)}\left|A_{i j}^{\alpha \beta}(y)-A_{i j}^{\alpha \beta}(z)\right| d y d z, \\
A_{R}^{\#}=\sup _{x \in \mathbb{R}^{d}} \sup _{r \leq R} \sum_{\alpha, \beta=1}^{d} \sum_{i, j=1}^{m} \operatorname{osc}\left(A_{i j}^{\alpha \beta}, B_{r}(x)\right) .
\end{gathered}
$$

Assumption 2.3. There is an increasing continuous function $\omega(r)$ defined on $[0, \infty)$ such that $\omega(0)=0$ and $A_{R}^{\#} \leq \omega(R)$.

Now we state our main results. In Theorem $2.4-2.7$, we use the notation $\boldsymbol{f}=$ $\left(f^{1}, \ldots, f^{m}\right)^{T}, \boldsymbol{g}=\left(\boldsymbol{g}_{\alpha}\right), \boldsymbol{g}_{\alpha}=\left(g_{\alpha}^{1}, \ldots, g_{\alpha}^{m}\right)^{T}, \alpha=1, \ldots, d$.

THEOREM 2.4. Let $1<p \leq q<\infty, 0<T<\infty$, and the coefficient matrices of $P$ satisfy Assumption 2.1 and 2.3. Then for any $f \in L_{q, p}\left((0, T) \times \mathbb{R}^{d}\right)$, there exists a unique $\boldsymbol{u} \in W_{q, p}^{1,2}\left((0, T) \times \mathbb{R}^{d}\right)$ such that $P \boldsymbol{u}=\boldsymbol{f}$ in $(0, T) \times \mathbb{R}^{d}$ and $\boldsymbol{u}(0, \cdot)=0$. Furthermore, there is a constant $N$, depending only on $d, m, p, q, \delta, K, T$, and the function $\omega$, such that

$$
\|\boldsymbol{u}\|_{W_{q, p}^{1,2}\left((0, T) \times \mathbb{R}^{d}\right)} \leq N\|P \boldsymbol{u}\|_{L_{q, p}\left((0, T) \times \mathbb{R}^{d}\right)}
$$

for any $\boldsymbol{u} \in W_{q, p}^{1,2}\left((0, T) \times \mathbb{R}^{d}\right)$ satisfying $\boldsymbol{u}(0, \cdot)=0$.

Theorem 2.5. Let $p, q \in(1, \infty), T \in(0, \infty)$, and the coefficient matrices of $\mathcal{P}$ satisfy Assumption 2.1 and 2.3. Then for any $\boldsymbol{f}, \boldsymbol{g}_{\alpha} \in L_{q, p}\left((0, T) \times \mathbb{R}^{d}\right)$, there exists a unique $\boldsymbol{u} \in \mathcal{H}_{q, p}^{1}\left((0, T) \times \mathbb{R}^{d}\right)$ such that $\mathcal{P} \boldsymbol{u}=\boldsymbol{f}+D_{\alpha} \boldsymbol{g}_{\alpha}$ in $(0, T) \times \mathbb{R}^{d}$ and $\boldsymbol{u}(0, \cdot)=0$. Furthermore, there is a constant $N$, depending only on $d, m, p, q, \delta, K, T$, and the function $\omega$, such that

$$
\|\boldsymbol{u}\|_{\mathcal{H}_{q, p}^{1}\left((0, T) \times \mathbb{R}^{d}\right)} \leq N\left(\|\boldsymbol{f}\|_{L_{q, p}\left((0, T) \times \mathbb{R}^{d}\right)}+\|\boldsymbol{g}\|_{L_{q, p}\left((0, T) \times \mathbb{R}^{d}\right)}\right) .
$$

TheOREm 2.6. Let $p \in(1, \infty)$, and the coefficient matrices of L satisfy Assumption 2.1 and 2.3. Then there exist constants $\lambda_{0} \geq 0$ and $N$, depending only on $d, m$, $p, \delta, K$, and the function $\omega$, such that

$$
\lambda\|\boldsymbol{u}\|_{L_{p}\left(\mathbb{R}^{d}\right)}+\sqrt{\lambda}\|D \boldsymbol{u}\|_{L_{p}\left(\mathbb{R}^{d}\right)}+\left\|D^{2} \boldsymbol{u}\right\|_{L_{p}\left(\mathbb{R}^{d}\right)} \leq N\|L \boldsymbol{u}-\lambda \boldsymbol{u}\|_{L_{p}\left(\mathbb{R}^{d}\right)}
$$


holds for any $\boldsymbol{u} \in W_{p}^{2}\left(\mathbb{R}^{d}\right)$ and $\lambda \geq \lambda_{0}$. Moreover, for any $\lambda>\lambda_{0}$ and $\boldsymbol{f} \in L_{p}\left(\mathbb{R}^{d}\right)$, there exists a unique $\boldsymbol{u} \in W_{p}^{2}\left(\mathbb{R}^{d}\right)$ satisfying

$$
L \boldsymbol{u}-\lambda \boldsymbol{u}=\boldsymbol{f} \text { in } \quad \mathbb{R}^{d} .
$$

Theorem 2.7. Let $p \in(1, \infty)$ and the coefficient matrices of $\mathcal{L}$ satisfy Assumption 2.1 and 2.3. Then there exist constants $\lambda_{0} \geq 0$ and $N$, depending only on $d, m$, $p, \delta, K$, the function $\omega$, such that

$$
\lambda\|\boldsymbol{u}\|_{L_{p}\left(\mathbb{R}^{d}\right)}+\sqrt{\lambda}\|D \boldsymbol{u}\|_{L_{p}\left(\mathbb{R}^{d}\right)} \leq N(\sqrt{\lambda}+1)\|\mathcal{L} \boldsymbol{u}-\lambda \boldsymbol{u}\|_{W_{p}^{-1}\left(\mathbb{R}^{d}\right)}
$$

holds for any $\boldsymbol{u} \in W_{p}^{1}\left(\mathbb{R}^{d}\right)$ and $\lambda \geq \lambda_{0}$. Moreover, for any $\boldsymbol{f}, \boldsymbol{g}_{\alpha} \in L_{p}\left(\mathbb{R}^{d}\right)$ and $\lambda>\lambda_{0}$, there exists a unique $\boldsymbol{u} \in W_{p}^{1}\left(\mathbb{R}^{d}\right)$ such that

$$
\mathcal{L} \boldsymbol{u}-\lambda \boldsymbol{u}=\boldsymbol{f}+D_{\alpha} \boldsymbol{g}_{\alpha} \quad \text { in } \quad \mathbb{R}^{d} .
$$

3. Preliminary results. Throughout this section we set

$$
\bar{P} \boldsymbol{u}=-\boldsymbol{u}_{t}+A^{\alpha \beta} D_{\alpha \beta} \boldsymbol{u},
$$

where the entries of coefficient matrices $A^{\alpha \beta}$ are measurable functions of only $t \in \mathbb{R}$, i.e., $A_{i j}^{\alpha \beta}=A_{i j}^{\alpha \beta}(t)$, satisfying Assumption 2.1. With this operator $\bar{P}$ we have the following theorem, which is proved in section 4 , first for the case $p=2$, and then for the general case $1<p<\infty$.

Theorem 3.1. Let $\boldsymbol{u} \in W_{p}^{1,2}\left(\mathbb{R}_{T}^{d+1}\right), T \in(-\infty, \infty]$. Then there exists a constant $N=N(d, m, p, \delta, K)$ such that

$$
\begin{aligned}
\lambda\|\boldsymbol{u}\|_{L_{p}\left(\mathbb{R}_{T}^{d+1}\right)}+\sqrt{\lambda}\|D \boldsymbol{u}\|_{L_{p}\left(\mathbb{R}_{T}^{d+1}\right)}+\left\|D^{2} \boldsymbol{u}\right\|_{L_{p}\left(\mathbb{R}_{T}^{d+1}\right)} & \\
& +\left\|\boldsymbol{u}_{t}\right\|_{L_{p}\left(\mathbb{R}_{T}^{d+1}\right)} \leq N\|\bar{P} \boldsymbol{u}-\lambda \boldsymbol{u}\|_{L_{p}\left(\mathbb{R}_{T}^{d+1}\right)}
\end{aligned}
$$

for all $\lambda \geq 0$. Moreover, for any $\lambda>0$ and $\boldsymbol{f} \in L_{p}\left(\mathbb{R}_{T}^{d+1}\right)$, there exists a unique $\boldsymbol{u} \in W_{p}^{1,2}\left(\mathbb{R}_{T}^{d+1}\right)$ satisfying $\bar{P} \boldsymbol{u}-\lambda \boldsymbol{u}=\boldsymbol{f}$.

The above theorem, along with the arguments in [23], allows us to obtain the following lemmas and theorems.

Lemma 3.2. Let $0<r<R<\infty$ and $\boldsymbol{u} \in W_{p, l o c}^{1,2}\left(\mathbb{R}^{d+1}\right)$. Then

$$
\left\|\boldsymbol{u}_{t}\right\|_{L_{p}\left(Q_{r}\right)}+\left\|D^{2} \boldsymbol{u}\right\|_{L_{p}\left(Q_{r}\right)} \leq N\left(\|\bar{P} \boldsymbol{u}\|_{L_{p}\left(Q_{R}\right)}+\|D \boldsymbol{u}\|_{L_{p}\left(Q_{R}\right)}+\|\boldsymbol{u}\|_{L_{p}\left(Q_{R}\right)}\right),
$$

where $N=N(d, m, p, \delta, K, r, R)$.

Proof. See the proof of Lemma 5.2 in [23].

Lemma 3.3. Let $0<r<R<\infty$ and $\boldsymbol{u} \in C_{\text {loc }}^{\infty}\left(\mathbb{R}^{d+1}\right)$. Assume that $\bar{P} \boldsymbol{u}=0$ in $Q_{R}$. Then for any multi-index $\gamma$, we have

$$
\sup _{Q_{r}}\left|D^{\gamma} \boldsymbol{u}\right|+\sup _{Q_{r}}\left|D^{\gamma} \boldsymbol{u}_{t}\right| \leq N\left(\|D \boldsymbol{u}\|_{L_{p}\left(Q_{R}\right)}+\|\boldsymbol{u}\|_{L_{p}\left(Q_{R}\right)}\right),
$$

where $N=N(|\gamma|, d, m, \delta, p, K, r, R)$. 
Proof. See the proof of Lemma 5.8 in [23].

Lemma 3.4. Let $\boldsymbol{u} \in C_{l o c}^{\infty}\left(\mathbb{R}^{d+1}\right)$ and $\lambda \geq 0$. Assume that $\bar{P} \boldsymbol{u}-\lambda \boldsymbol{u}=0$ in $Q_{2}$. Then for any multi-index $\gamma$, we have

$$
\begin{gathered}
\sup _{Q_{1}}\left|D^{\gamma}\left(D^{2} \boldsymbol{u}\right)\right|+\sup _{Q_{1}}\left|D^{\gamma} \boldsymbol{u}_{t}\right| \leq N\left(\left\|\boldsymbol{u}_{t}\right\|_{L_{p}\left(Q_{2}\right)}+\left\|D^{2} \boldsymbol{u}\right\|_{L_{p}\left(Q_{2}\right)}+\sqrt{\lambda}\|D \boldsymbol{u}\|_{L_{p}\left(Q_{2}\right)}\right), \\
\sup _{Q_{1}}\left|D^{\gamma}(D \boldsymbol{u})\right|+\sup _{Q_{1}}\left|D^{\gamma} \boldsymbol{u}_{t}\right| \leq N\left(\|D \boldsymbol{u}\|_{L_{p}\left(Q_{2}\right)}+\sqrt{\lambda}\|\boldsymbol{u}\|_{L_{p}\left(Q_{2}\right)}\right)
\end{gathered}
$$

where $N=N(|\gamma|, d, m, p, \delta, K)$.

Proof. See the proof of Lemma 5.9 and 7.3 in [23].

TheOREm 3.5. Let $\lambda \geq 0, \kappa \geq 2$, and $r \in(0, \infty)$. Let $\boldsymbol{u} \in C_{\text {loc }}^{\infty}\left(\mathbb{R}^{d+1}\right)$ such that $\bar{P} \boldsymbol{u}-\lambda \boldsymbol{u}=0$ in $Q_{\kappa r}$. Then there is a constant $N$, depending only on $d, m, p, \delta$, and $K$, such that

$$
\begin{gathered}
f_{Q_{r}}\left|D^{2} \boldsymbol{u}(t, x)-\left(D^{2} \boldsymbol{u}\right)_{Q_{r}}\right|^{p} d x d t \leq N \kappa^{-p}\left(\left|D^{2} \boldsymbol{u}\right|^{p}+\lambda^{p / 2}|D \boldsymbol{u}|^{p}\right)_{Q_{\kappa r}} \\
f_{Q_{r}}\left|D \boldsymbol{u}(t, x)-(D \boldsymbol{u})_{Q_{r}}\right|^{p} d x d t \leq N \kappa^{-p}\left(|D \boldsymbol{u}|^{p}+\lambda^{p / 2}|\boldsymbol{u}|^{p}\right)_{Q_{\kappa r}}
\end{gathered}
$$

Proof. See the proof of Theorem 5.10 and 7.4 in [23].

By using the results above, one can obtain the following estimates, which are important in proving the main theorems. For their proofs, we refer to Theorem 5.1 and 7.1 in [23].

THEOREM 3.6. There is a constant $N=N(d, m, p, \delta, K)$ such that, for $\boldsymbol{u} \in$ $W_{p, l o c}^{1,2}\left(\mathbb{R}^{d+1}\right), r \in(0, \infty)$, and $\kappa \geq 4$,

$$
f_{Q_{r}}\left|D^{2} \boldsymbol{u}(t, x)-\left(D^{2} \boldsymbol{u}\right)_{Q_{r}}\right|{ }^{p} d x d t \leq N \kappa^{d+2}\left(|\bar{P} \boldsymbol{u}|^{p}\right)_{Q_{\kappa r}}+N \kappa^{-p}\left(\left|D^{2} \boldsymbol{u}\right|^{p}\right)_{Q_{\kappa r}} .
$$

TheOrem 3.7. Let $\boldsymbol{u} \in \mathcal{H}_{p, l o c}^{1}\left(\mathbb{R}^{d+1}\right), \boldsymbol{g}=\left(\boldsymbol{g}_{\alpha}\right) \in L_{p, l o c}\left(\mathbb{R}^{d+1}\right), r \in(0, \infty)$, and $\kappa \geq 4$. Assume $\bar{P} \boldsymbol{u}=D_{\alpha} \boldsymbol{g}_{\alpha}$ in $Q_{\kappa r}$. Then there is a constant $N=N(d, m, p, \delta, K)$ such that,

$$
f_{Q_{r}}\left|D \boldsymbol{u}(t, x)-(D \boldsymbol{u})_{Q_{r}}\right|^{p} d x d t \leq N \kappa^{d+2}\left(|\boldsymbol{g}|^{p}\right)_{Q_{\kappa r}}+N \kappa^{-p}\left(|D \boldsymbol{u}|^{p}\right)_{Q_{\kappa r}} .
$$

REMARK 3.8. It is worth noting that Theorem 3.6 and 3.7 can be improved. Indeed, due to Lemma 6.2 and 8.2, following the proofs of Theorem 5.1 and 7.1 in [23] one can actually get

$$
f_{Q_{r}}\left|D^{2} \boldsymbol{u}(t, x)-\left(D^{2} \boldsymbol{u}\right)_{Q_{r}}\right|^{p} d x d t \leq N \kappa^{d+2}\left(|\bar{P} \boldsymbol{u}|^{p}\right)_{Q_{\kappa r}}+N \kappa^{-p}\left(\left|D^{2} \boldsymbol{u}\right|\right)_{Q_{\kappa r}}^{p}
$$

under the assumptions of Theorem 3.6, and

$$
f_{Q_{r}}\left|D \boldsymbol{u}(t, x)-(D \boldsymbol{u})_{Q_{r}}\right|^{p} d x d t \leq N \kappa^{d+2}\left(|\boldsymbol{g}|^{p}\right)_{Q_{\kappa r}}+N \kappa^{-p}(|D \boldsymbol{u}|)_{Q_{\kappa r}}^{p}
$$

under the assumptions of Theorem 3.7. 
4. Systems with coefficients independent of the spatial variables. In this section we prove Theorem 3.1, so recall that the operator $\bar{P}$ was defined in (4), where $A^{\alpha \beta}$ are assumed to be matrices of measurable functions depending only on $t \in \mathbb{R}$, i.e., $A_{i j}^{\alpha \beta}=A_{i j}^{\alpha \beta}(t)$. Theorem 3.1 , in case $p=2$, is easily proved using Fourier transform methods.

Proof of Theorem 3.1 when $p=2$. It is enough to prove the estimate (5) for $\lambda>0$ and $\boldsymbol{u} \in C_{c}^{\infty}\left(\mathbb{R}^{d+1}\right)$. Indeed, the estimate (5) for $\lambda=0$ is proved by letting $\lambda \searrow 0$ once the estimate is established for $\lambda>0$. To obtain the second assertion (solvability) of the theorem, we can just use the estimate (5), the solvability of the heat equation (i.e., $A_{i j}^{\alpha \beta}=\delta_{\alpha \beta} \delta_{i j}$ ), and the method of continuity.

For $\boldsymbol{u} \in C_{c}^{\infty}\left(\mathbb{R}^{d+1}\right)$, set $\boldsymbol{f}=\bar{P} \boldsymbol{u}-\lambda \boldsymbol{u}$. Then by taking Fourier transforms in $x$-variables we see

$$
-\widetilde{\boldsymbol{u}}_{t}-\xi_{\alpha} \xi_{\beta} A^{\alpha \beta} \widetilde{\boldsymbol{u}}-\lambda \widetilde{\boldsymbol{u}}=\widetilde{\boldsymbol{f}}
$$

where $\widetilde{\boldsymbol{u}}=\left(\widetilde{u}^{1}, \cdots, \widetilde{u}^{m}\right)^{T}$ and $\widetilde{u}^{i}(t, \xi), i=1, \cdots, m$, are the Fourier transforms of $u^{i}$ with respect to the spatial variables. By multiplying both sides of the above equation from the left by the complex conjugate of the Fourier transform of $(\lambda-\Delta) \boldsymbol{u}^{T}$, we obtain

$$
-\left(\lambda+|\xi|^{2}\right) \overline{\widetilde{u}} \cdot \widetilde{\boldsymbol{u}_{t}}-\left(\lambda+|\xi|^{2}\right) \xi_{\alpha} \xi_{\beta} \overline{\widetilde{\boldsymbol{u}}^{T}} A^{\alpha \beta} \widetilde{\boldsymbol{u}}-\lambda\left(\lambda+|\xi|^{2}\right) \overline{\widetilde{\boldsymbol{u}}} \cdot \widetilde{\boldsymbol{u}}=\left(\lambda+|\xi|^{2}\right) \overline{\widetilde{\boldsymbol{u}}} \cdot \tilde{\boldsymbol{f}}
$$

Note that

$$
\begin{aligned}
\Re\left(\overline{\widetilde{u}} \cdot \widetilde{\boldsymbol{u}}_{t}\right)= & \frac{1}{2} \frac{\partial}{\partial t}|\widetilde{\boldsymbol{u}}|^{2}, \quad \int_{-\infty}^{T} \frac{\partial}{\partial t}|\widetilde{\boldsymbol{u}}(t, \xi)|^{2} d t=|\widetilde{\boldsymbol{u}}(T, \xi)|^{2}, \\
\Re\left(\xi_{\alpha} \xi_{\beta} \overline{\widetilde{\boldsymbol{u}}^{T}} A^{\alpha \beta} \widetilde{\boldsymbol{u}}\right) & =\xi_{\alpha} \xi_{\beta} \Re\left(\widetilde{u}^{i}\right) A_{i j}^{\alpha \beta} \Re\left(\widetilde{u}^{j}\right)+\xi_{\alpha} \xi_{\beta} \Im\left(\widetilde{u}^{i}\right) A_{i j}^{\alpha \beta} \Im\left(\widetilde{u}^{j}\right) \\
& \geq \delta|\xi|^{2}|\widetilde{\boldsymbol{u}}|^{2} .
\end{aligned}
$$

Thus by taking the real parts of the equation (6) and integrating them, we have

$$
\delta \int_{\mathbb{R}_{T}^{d+1}}\left(\lambda+|\xi|^{2}\right)^{2}|\widetilde{\boldsymbol{u}}|^{2} d \xi d t \leq-\int_{\mathbb{R}_{T}^{d+1}}\left(\lambda+|\xi|^{2}\right) \Re(\overline{\widetilde{\boldsymbol{u}}} \cdot \widetilde{\boldsymbol{f}}) d \xi d t
$$

We see that

$$
-\left(\lambda+|\xi|^{2}\right) \Re(\overline{\widetilde{\boldsymbol{u}}} \cdot \widetilde{\boldsymbol{f}}) \leq\left(\lambda+|\xi|^{2}\right)\left(\varepsilon|\widetilde{\boldsymbol{u}}|^{2}+\left.\frac{1}{4 \varepsilon} \widetilde{\mid \boldsymbol{f}}\right|^{2}\right)
$$

for any $\varepsilon>0$. Set $\varepsilon=\delta\left(\lambda+|\xi|^{2}\right) / 2$, then

$$
\begin{gathered}
-\int_{\mathbb{R}_{T}^{d+1}}\left(\lambda+|\xi|^{2}\right) \Re(\overline{\widetilde{\boldsymbol{u}}} \cdot \widetilde{\boldsymbol{f}}) d \xi d t \leq \delta / 2 \int_{\mathbb{R}_{T}^{d+1}}\left(\lambda+|\xi|^{2}\right)^{2}|\widetilde{\boldsymbol{u}}|^{2} d \xi d t \\
+(2 \delta)^{-1} \int_{\mathbb{R}_{T}^{d+1}}|\widetilde{\boldsymbol{f}}|^{2} d \xi d t
\end{gathered}
$$

From this and (7) it follows that

$$
\int_{\mathbb{R}_{T}^{d+1}}\left(\lambda+|\xi|^{2}\right)^{2}|\widetilde{\boldsymbol{u}}|^{2} d \xi d t \leq \delta^{-2} \int_{\mathbb{R}_{T}^{d+1}}|\widetilde{\boldsymbol{f}}|^{2} d \xi d t .
$$


This along with Plancherel's theorem implies the inequality (5).

Let $\mathcal{Q}=\left\{Q_{r}(t, x):(t, x) \in \mathbb{R}^{d+1}, r \in(0, \infty)\right\}$. For a function $g$ defined on $\mathbb{R}^{d+1}$, we denote its (parabolic) maximal and sharp function, respectively, by

$$
\begin{aligned}
M g(t, x) & =\sup _{Q \in \mathcal{Q}:(t, x) \in Q} f_{Q}|g(s, y)| d y d s, \\
g^{\#}(t, x) & =\sup _{Q \in \mathcal{Q}:(t, x) \in Q} f_{Q}\left|g(s, y)-(g)_{Q}\right| d y d s .
\end{aligned}
$$

Now that Theorem 3.1 is proved for the case $p=2$, the results in section 3 are all available if $p=2$. Then we proceed as follows to obtain the $L_{p}$-estimate, $1<p<\infty$, for the parabolic system in (4).

Proposition 4.1. There exists a constant $N=N(d, m, p, \delta, K)$ such that, for any $u \in C_{c}^{\infty}\left(\mathbb{R}^{d+1}\right)$, we have

$$
\left\|D^{2} \boldsymbol{u}\right\|_{L_{p}\left(\mathbb{R}^{d+1}\right)} \leq N\|\bar{P} \boldsymbol{u}\|_{L_{p}\left(\mathbb{R}^{d+1}\right)} .
$$

Proof. Recall that the case $p=2$ is proved above, so we first consider the case $p>2$. From Theorem 3.6 along with an appropriate translation, we have

$$
\begin{aligned}
\left(\left|D^{2} \boldsymbol{u}-\left(D^{2} \boldsymbol{u}\right)_{Q_{r}\left(t_{0}, x_{0}\right)}\right|^{2}\right)_{Q_{r}\left(t_{0}, x_{0}\right)} & \\
\leq & N \kappa^{d+2}\left(|\bar{P} \boldsymbol{u}|^{2}\right)_{Q_{\kappa r}\left(t_{0}, x_{0}\right)}+N \kappa^{-2}\left(\left|D^{2} \boldsymbol{u}\right|^{2}\right)_{Q_{\kappa r}\left(t_{0}, x_{0}\right)}
\end{aligned}
$$

for $\left(t_{0}, x_{0}\right) \in \mathbb{R}^{d+1}, r>0$ and $\kappa \geq 4$. Let

$$
\mathcal{A}(t, x)=M\left(|\bar{P} \boldsymbol{u}|^{2}\right)(t, x), \quad \mathcal{B}(t, x)=M\left(\left|D^{2} \boldsymbol{u}\right|^{2}\right)(t, x) .
$$

Then $\left(|\bar{P} \boldsymbol{u}|^{2}\right)_{Q_{\kappa r}\left(t_{0}, x_{0}\right)} \leq \mathcal{A}(t, x)$ for all $(t, x) \in Q_{r}\left(t_{0}, x_{0}\right)$. Similar inequalities are obtained for $\mathcal{B}$. From this and (8) it follows that, for any $(t, x) \in \mathbb{R}^{d+1}$ and $Q \in \mathcal{Q}$ such that $(t, x) \in Q$,

$$
\left(\left|D^{2} \boldsymbol{u}-\left(D^{2} \boldsymbol{u}\right)_{Q}\right|^{2}\right)_{Q} \leq N \kappa^{d+2} \mathcal{A}(t, x)+N \kappa^{-2} \mathcal{B}(t, x)
$$

for $\kappa \geq 4$. Take the supremum of the left-hand side of the above inequality over all $Q \in \mathcal{Q}$ containing $(t, x)$. Also observe that

$$
\left(\left|D^{2} \boldsymbol{u}-\left(D^{2} \boldsymbol{u}\right)_{Q}\right|\right)_{Q}^{2} \leq\left(\left|D^{2} \boldsymbol{u}-\left(D^{2} \boldsymbol{u}\right)_{Q}\right|^{2}\right)_{Q} .
$$

Then we obtain

$$
\left(\left(D^{2} \boldsymbol{u}\right)^{\#}(t, x)\right)^{2} \leq N \kappa^{d+2} \mathcal{A}(t, x)+N \kappa^{-2} \mathcal{B}(t, x)
$$

for $\kappa \geq 4$, where $N=N(d, m, \delta, K)$. Apply the Fefferman-Stein theorem on sharp functions and the Hardy-Littlewood maximal function theorem on the above inequality. More precisely,

$$
\begin{aligned}
\left\|D^{2} \boldsymbol{u}\right\|_{L_{p}\left(\mathbb{R}^{d+1}\right)} & \leq N\left\|\left(D^{2} \boldsymbol{u}\right)^{\#}\right\|_{L_{p}\left(\mathbb{R}^{d+1}\right)} \\
& \leq N \kappa^{(d+2) / 2}\left\|M\left(|\bar{P} \boldsymbol{u}|^{2}\right)\right\|_{L_{p / 2}\left(\mathbb{R}^{d+1}\right)}^{1 / 2}+N \kappa^{-1}\left\|M\left(\left|D^{2} \boldsymbol{u}\right|^{2}\right)\right\|_{L_{p / 2}\left(\mathbb{R}^{d+1}\right)}^{1 / 2} \\
& \leq N \kappa^{(d+2) / 2}\|\bar{P} \boldsymbol{u}\|_{L_{p}\left(\mathbb{R}^{d+1}\right)}+N \kappa^{-1}\left\|D^{2} \boldsymbol{u}\right\|_{L_{p}\left(\mathbb{R}^{d+1}\right)},
\end{aligned}
$$


where the last inequality is possible due to the assumption that $p>2$. Now choose a large enough $\kappa$, then we see that the inequality in the theorem follows if $p>2$. For the case $1<p<2$, we use the duality argument. $\mathrm{Q}$

Let us complete the proof of Theorem 3.1 for all $1<p<\infty$.

Proof. [Proof of Theorem 3.1] First set $T=\infty$. As in the case $p=2$, it is enough to prove the estimate in the theorem, which follows from the estimate proved in Proposition 4.1 and Agmon's idea described in the proof of Theorem 4.1 in [22]. For the case $T<\infty$, we make use of the argument in the proof of Theorem 2.1 in [22], i.e. by using the fact that $\boldsymbol{u}=\boldsymbol{w}$ for $t<T$ where $\boldsymbol{w} \in W_{p}^{1,2}\left(\mathbb{R}^{d+1}\right)$ solves $\bar{P} \boldsymbol{w}-\lambda \boldsymbol{w}=I_{t<T}(\bar{P} \boldsymbol{u}-\lambda \boldsymbol{u})$. The theorem is proved.

5. Systems in non-divergence form with $\mathrm{VMO}_{x}$ coefficients in $L_{p}$. In this section we assume that the coefficients $A_{i j}^{\alpha \beta}(t, x)$ are measurable in $t \in \mathbb{R}$ and VMO in $x \in \mathbb{R}^{d}$. More precisely, Assumption 2.3 as well as Assumption 2.1 are satisfied.

Here is the main result of this section.

TheOREm 5.1. Let $\boldsymbol{u} \in W_{p}^{1,2}\left(\mathbb{R}_{T}^{d+1}\right), T \in(-\infty, \infty]$. Then there exist constants $\lambda_{0} \geq 0$ and $N$, depending only on $d, m, p, \delta, K$, and the function $\omega$, such that

$$
\begin{aligned}
\lambda\|\boldsymbol{u}\|_{L_{p}\left(\mathbb{R}_{T}^{d+1}\right)}+\sqrt{\lambda}\|D \boldsymbol{u}\|_{L_{p}\left(\mathbb{R}_{T}^{d+1}\right)}+\left\|D^{2} \boldsymbol{u}\right\|_{L_{p}\left(\mathbb{R}_{T}^{d+1}\right)} & \\
& +\left\|\boldsymbol{u}_{t}\right\|_{L_{p}\left(\mathbb{R}_{T}^{d+1}\right)} \leq N\|P \boldsymbol{u}-\lambda \boldsymbol{u}\|_{L_{p}\left(\mathbb{R}_{T}^{d+1}\right)}
\end{aligned}
$$

for all $\lambda \geq \lambda_{0}$. Moreover, for any $\lambda>\lambda_{0}$ and $\boldsymbol{f} \in L_{p}\left(\mathbb{R}_{T}^{d+1}\right)$, there exists a unique $\boldsymbol{u} \in W_{p}^{1,2}\left(\mathbb{R}_{T}^{d+1}\right)$ satisfying $P \boldsymbol{u}-\lambda \boldsymbol{u}=\boldsymbol{f}$.

Remark 5.2. This theorem implies Theorem 2.4 for $p=q$. This is justified again by using the argument in the proof of Theorem 2.1 in [22].

To prove the above theorem, we need some preliminary results presented below. First recall that we have proved Theorem 3.1 in section 4 , so the results in section 3 are available for all $p \in(1, \infty)$. Then we have

Lemma 5.3. Let $B^{\alpha}=C=0$. Also let $\mu, \nu \in(1, \infty), 1 / \mu+1 / \nu=1$, and $R \in(0, \infty)$. Then there exists a constant $N=N(d, m, q, \delta, K, \mu)$ such that, for any $\boldsymbol{u} \in C_{c}^{\infty}\left(\mathbb{R}^{d+1}\right)$ vanishing outside $Q_{R}$, we have

$$
\begin{aligned}
& \left(\left|D^{2} \boldsymbol{u}-\left(D^{2} \boldsymbol{u}\right)_{Q_{r}\left(t_{0}, x_{0}\right)}\right|^{q}\right)_{Q_{r}\left(t_{0}, x_{0}\right)} \leq N \kappa^{-q}\left(\left|D^{2} \boldsymbol{u}\right|^{q}\right)_{Q_{\kappa r}\left(t_{0}, x_{0}\right)} \\
& +N \kappa^{d+2}\left(\left(|P \boldsymbol{u}|^{q}\right)_{Q_{\kappa r}\left(t_{0}, x_{0}\right)}+\omega(R)^{1 / \nu}\left(\left|D^{2} \boldsymbol{u}\right|^{q \mu}\right)_{Q_{\kappa r}\left(t_{0}, x_{0}\right)}^{1 / \mu}\right)
\end{aligned}
$$

for $r \in(0, \infty), \kappa \geq 4$, and $\left(t_{0}, x_{0}\right) \in \mathbb{R}^{d+1}$.

Proof. Let $\kappa \geq 4$ and $r \in(0, \infty)$. We introduce another coefficients $\bar{A}^{\alpha \beta}$ defined as follows.

$$
\begin{aligned}
& \bar{A}^{\alpha \beta}(t)=f_{B_{\kappa r}\left(t_{0}, x_{0}\right)} A^{\alpha \beta}(t, y) d y \quad \text { if } \quad \kappa r<R, \\
& \bar{A}^{\alpha \beta}(t)=f_{B_{R}} A^{\alpha \beta}(t, y) d y \quad \text { if } \quad \kappa r \geq R .
\end{aligned}
$$


Set $\bar{P} \boldsymbol{u}=-\boldsymbol{u}_{t}+\bar{A}^{\alpha \beta} D_{\alpha \beta} \boldsymbol{u}$. Then from Theorem 3.6 (also using a translation) it follows that

$$
\begin{aligned}
&\left(\left|D^{2} \boldsymbol{u}-\left(D^{2} \boldsymbol{u}\right)_{Q_{r}\left(t_{0}, x_{0}\right)}\right|^{q}\right)_{Q_{r}\left(t_{0}, x_{0}\right)} \\
& \leq N \kappa^{d+2}\left(|\bar{P} \boldsymbol{u}|^{q}\right)_{Q_{\kappa r}\left(t_{0}, x_{0}\right)}+N \kappa^{-q}\left(\left|D^{2} \boldsymbol{u}\right|^{q}\right)_{Q_{\kappa r}\left(t_{0}, x_{0}\right)} .
\end{aligned}
$$

Note that

$$
\int_{Q_{\kappa r}\left(t_{0}, x_{0}\right)}|\bar{P} \boldsymbol{u}|^{q} d x d t \leq N \int_{Q_{\kappa r}\left(t_{0}, x_{0}\right)}|P \boldsymbol{u}|^{q} d x d t+N \chi
$$

where

$$
\begin{aligned}
\chi= & \int_{Q_{\kappa r}\left(t_{0}, x_{0}\right)}\left|\left(\bar{A}^{\alpha \beta}-A^{\alpha \beta}\right) D_{\alpha \beta} \boldsymbol{u}\right|^{q} d x d t=\int_{Q_{\kappa r}\left(t_{0}, x_{0}\right) \cap Q_{R}}\left|\left(\bar{A}^{\alpha \beta}-A^{\alpha \beta}\right) D_{\alpha \beta} \boldsymbol{u}\right|^{q} d x d t \\
& \leq\left(\int_{Q_{\kappa r}\left(t_{0}, x_{0}\right) \cap Q_{R}}|\bar{A}-A|^{q \nu}\right)^{1 / \nu}\left(\int_{Q_{\kappa r}\left(t_{0}, x_{0}\right) \cap Q_{R}}\left|D^{2} \boldsymbol{u}\right|^{q \mu}\right)^{1 / \mu}:=I^{1 / \nu} J^{1 / \mu} .
\end{aligned}
$$

Using the definition of $\bar{A}^{\alpha \beta}$ and assumptions on $A^{\alpha \beta}$, we obtain the following estimates for $I$. If $\kappa r<R$,

$$
\begin{aligned}
I & \leq N \int_{t_{0}-(\kappa r)^{2}}^{t_{0}} \int_{B_{\kappa r}\left(x_{0}\right)}|\bar{A}-A| d x d t \\
& \leq N(\kappa r)^{d+2} \omega(\kappa r) \leq N(\kappa r)^{d+2} \omega(R) .
\end{aligned}
$$

In case $\kappa r \geq R$,

$$
I \leq N \int_{-R^{2}}^{0} \int_{B_{R}}|\bar{A}-A| d x d t \leq N R^{d+2} \omega(R) \leq N(\kappa r)^{d+2} \omega(R) .
$$

From the inequality (10) and the estimates for $I$, it follows that

$$
\left(|\bar{P} \boldsymbol{u}|^{q}\right)_{Q_{\kappa r}\left(t_{0}, x_{0}\right)} \leq N \omega(R)^{1 / \nu}\left(\left|D^{2} \boldsymbol{u}\right|^{q \mu}\right)_{Q_{\kappa r}\left(t_{0}, x_{0}\right)}^{1 / \mu}+N\left(|P \boldsymbol{u}|^{q}\right)_{Q_{\kappa r}\left(t_{0}, x_{0}\right)} .
$$

This, together with (9), gives us

$$
\begin{aligned}
&\left(\left|D^{2} \boldsymbol{u}-\left(D^{2} \boldsymbol{u}\right)_{Q_{r}\left(t_{0}, x_{0}\right)}\right|^{q}\right)_{Q_{r}\left(t_{0}, x_{0}\right)} \leq N \kappa^{d+2} \omega(R)^{1 / \nu}\left(\left|D^{2} \boldsymbol{u}\right|^{q \mu}\right)_{Q_{\kappa r}\left(t_{0}, x_{0}\right)}^{1 / \mu} \\
&+N \kappa^{d+2}\left(|P \boldsymbol{u}|^{q}\right)_{Q_{\kappa r}\left(t_{0}, x_{0}\right)}+N \kappa^{-q}\left(\left|D^{2} \boldsymbol{u}\right|^{q}\right)_{Q_{\kappa r}\left(t_{0}, x_{0}\right)}
\end{aligned}
$$

for any $r>0$ and $\kappa \geq 4$. This finishes the proof.

Proposition 5.4. Let $B^{\alpha}=C=0$. Then there exist constants $R=$ $R(d, m, p, \delta, K, \omega)$ and $N=N(d, m, p, \delta, K)$ such that, for $\boldsymbol{u} \in C_{c}^{\infty}\left(\mathbb{R}^{d+1}\right)$ vanishing outside $Q_{R}$, we have

$$
\left\|D^{2} \boldsymbol{u}\right\|_{L_{p}\left(\mathbb{R}^{d+1}\right)} \leq N\|P \boldsymbol{u}\|_{L_{p}\left(\mathbb{R}^{d+1}\right)} .
$$

Proof. Let $\boldsymbol{u}$ be an infinitely differentiable function with compact support in $Q_{R}$, where $R$ will be chosen below. Take $q>1$ and $\mu>1$ such that $1<q \mu<p$. Let

$$
\mathcal{A}(t, x)=M\left(|P \boldsymbol{u}|^{q}\right)(t, x), \quad \mathcal{B}(t, x)=M\left(\left|D^{2} \boldsymbol{u}\right|^{q}\right)(t, x),
$$




$$
\mathcal{C}(t, x)=\left(M\left(\left|D^{2} \boldsymbol{u}\right|^{q \mu}\right)(t, x)\right)^{1 / \mu} .
$$

We note that $\left(|P \boldsymbol{u}|^{q}\right)_{Q_{k r}\left(t_{0}, x_{0}\right)} \leq \mathcal{A}(t, x)$ for all $(t, x) \in Q_{r}\left(t_{0}, x_{0}\right)$. Similar inequalities hold true for $\mathcal{B}$ and $\mathcal{C}$. Then by Lemma 5.3 it follows that (recall $1 / \mu+1 / \nu=1$ )

$$
\left(\left|D^{2} \boldsymbol{u}-\left(D^{2} \boldsymbol{u}\right)_{Q}\right|^{q}\right)_{Q} \leq N \kappa^{-q} \mathcal{B}(t, x)+N \kappa^{d+2}\left(\mathcal{A}(t, x)+\omega(R)^{1 / \nu} \mathcal{C}(t, x)\right)
$$

for all $\kappa \geq 4,(t, x) \in \mathbb{R}^{d+1}$, and $Q \in \mathcal{Q}$ such that $(t, x) \in Q$. Take the supremum of the left-hand side of the above inequality over all $Q \in \mathcal{Q}$ containing $(t, x)$. Also observe that

$$
\left(\left|D^{2} \boldsymbol{u}-\left(D^{2} \boldsymbol{u}\right)_{Q}\right|\right)_{Q}^{q} \leq\left(\left|D^{2} \boldsymbol{u}-\left(D^{2} \boldsymbol{u}\right)_{Q}\right|^{q}\right)_{Q}
$$

Then we obtain

$$
\left(\left(D^{2} \boldsymbol{u}\right)^{\#}(t, x)\right)^{q} \leq N \kappa^{d+2} \mathcal{A}(t, x)+N \kappa^{-q} \mathcal{B}(t, x)+N \kappa^{d+2} \omega(R)^{1 / \nu} \mathcal{C}(t, x)
$$

for all $\kappa \geq 4,(t, x) \in \mathbb{R}^{d+1}$, where $N=N(d, m, p, \delta, K)$. That is,

$$
\begin{aligned}
\left(D^{2} \boldsymbol{u}\right)^{\#}(t, x) \leq N \kappa^{(d+2) / q} \mathcal{A}(t, x)^{1 / q}+N \kappa^{-1} \mathcal{B}(t & x)^{1 / q} \\
& +N \kappa^{(d+2) / q} \omega(R)^{1 / q \nu} \mathcal{C}(t, x)^{1 / q} .
\end{aligned}
$$

Applying the Fefferman-Stein theorem on sharp functions and the Hardy-Littlewood maximal function theorem on the above inequality (recall $p>q \mu$ ), we have

$$
\begin{gathered}
\left\|D^{2} \boldsymbol{u}\right\|_{L_{p}\left(\mathbb{R}^{d+1}\right)} \leq N \kappa^{(d+2) / q}\left\|\mathcal{A}^{1 / q}\right\|_{L_{p}\left(\mathbb{R}^{d+1}\right)}+N \kappa^{-1}\left\|\mathcal{B}^{1 / q}\right\|_{L_{p}\left(\mathbb{R}^{d+1}\right)} \\
+N \kappa^{(d+2) / q} \omega(R)^{1 /(q \nu)}\left\|\mathcal{C}^{1 / q}\right\|_{L_{p}\left(\mathbb{R}^{d+1}\right)} \\
\leq N \kappa^{(d+2) / q}\|P \boldsymbol{u}\|_{L_{p}\left(\mathbb{R}^{d+1}\right)}+N\left(\kappa^{-1}+\kappa^{(d+2) / q} \omega(R)^{1 /(q \nu)}\right)\left\|D^{2} \boldsymbol{u}\right\|_{L_{p}\left(\mathbb{R}^{d+1}\right)},
\end{gathered}
$$

where $\kappa \geq 4$. Choose a big enough $\kappa$ and then a small enough $R$, so that

$$
N\left(\kappa^{-1}+\kappa^{(d+2) / q} \omega(R)^{1 /(q \nu)}\right)<1 / 2 .
$$

Then the estimate in the proposition follows.

Proof of Theorem 5.1. As noted earlier, it suffices to prove the estimate in the theorem. Thanks to Proposition 5.4, by using a partition of unity (see the proof of Theorem 5.7 in [22]), we obtain an estimate

$$
\left\|D^{2} \boldsymbol{u}\right\|_{L_{p}\left(\mathbb{R}^{d+1}\right)} \leq N\left(\|P \boldsymbol{u}\|_{L_{p}\left(\mathbb{R}^{d+1}\right)}+\|D \boldsymbol{u}\|_{L_{p}\left(\mathbb{R}^{d+1}\right)}+\|\boldsymbol{u}\|_{L_{p}\left(\mathbb{R}^{d+1}\right)}\right),
$$

where $N=N(d, m, p, \delta, K, \omega)$ (this inequality is possible without having the condition $\left.B^{\alpha}=C=0\right)$. Then using again Agmon's idea in the proof of Theorem 4.1 in [22] and choosing a sufficiently large $\lambda_{0}$, we arrive at the estimate in the theorem when $T=\infty$. To deal with the case $T<\infty$ we use again the argument in the proof of Theorem 2.1 in [22]. 
6. Proof of Theorem 2.4. Recall that by $P$ we mean the operator defined in (1), where the matrices $A^{\alpha \beta}, B^{\alpha}$, and $C$ satisfy Assumption 2.1. Especially, the matrices $A^{\alpha \beta}$ satisfy Assumption 2.3.

The following two lemmas are possible since we have the $L_{p}$-estimate of systems with $\mathrm{VMO}_{x}$ coefficients (see Theorem 5.1). Their proofs can be done by imitating the proofs of Lemma 6.3 and Corollary 6.4 in [23].

Lemma 6.1. Let $r \in(0,1], \kappa \in(1, \infty)$, and $\boldsymbol{u} \in W_{p, l o c}^{1,2}\left(\mathbb{R}^{d+1}\right)$. Then

$$
\left\|D^{2} \boldsymbol{u}\right\|_{L_{p}\left(Q_{r}\right)} \leq N\left(\|P \boldsymbol{u}\|_{L_{p}\left(Q_{\kappa r}\right)}+r^{-1}\|D \boldsymbol{u}\|_{L_{p}\left(Q_{\kappa r}\right)}+r^{-2}\|\boldsymbol{u}\|_{L_{p}\left(Q_{\kappa r}\right)}\right),
$$

where $N$, independent of $r \in(0,1]$, depends only on $\kappa, d, m, p, \delta, K$ and the function $\omega$.

Lemma 6.2. Let $p>q \geq 1$ and $r \in(0,1]$. Let $B^{\alpha}=C=0$ and assume that $\boldsymbol{u} \in W_{p, l o c}^{1,2}\left(\mathbb{R}^{d+1}\right)$ satisfies $P \boldsymbol{u}=0$ in $Q_{2 r}$. Then

$$
\left(\left|D^{2} \boldsymbol{u}\right|^{p}\right)_{Q_{r}}^{1 / p} \leq N\left(\left|D^{2} \boldsymbol{u}\right|\right)_{Q_{2 r}} \leq N\left(\left|D^{2} \boldsymbol{u}\right|^{q}\right)_{Q_{2 r}}^{1 / q}
$$

where $N$ depends only on $d, m, p, \delta, K$, and the function $\omega$.

In the following, in order to prove Theorem 2.4, we shall use the idea in [23]. However, since we have parabolic systems and our statements are slightly different from those in [23], we present here some proofs.

Theorem 6.3. Let $B^{\alpha}=C=0$. Then there exists a constant $N=$ $N(d, m, p, \delta, K, \omega)$ such that, for any $\boldsymbol{u} \in C_{c}^{\infty}\left(\mathbb{R}^{d+1}\right), \kappa \geq 8$, and $r \in(0,1 / \kappa]$, we have

$$
\begin{aligned}
f_{Q_{r}} \mid D^{2} \boldsymbol{u}- & \left.\left(D^{2} \boldsymbol{u}\right)_{Q_{r}}\right|^{p} d x d t \\
& \leq N \kappa^{d+2}\left(|P \boldsymbol{u}|^{p}\right)_{Q_{\kappa r}}+N\left(\kappa^{-p}+\kappa^{d+2} \omega(\kappa r)^{1 / 2}\right)\left(\left|D^{2} \boldsymbol{u}\right|^{p}\right)_{Q_{\kappa r}} .
\end{aligned}
$$

Proof. For given $\boldsymbol{u} \in C_{c}^{\infty}\left(\mathbb{R}^{d+1}\right), \kappa \geq 8$, and $r \in(0,1 / \kappa]$, find a unique function $\widetilde{\boldsymbol{w}} \in W_{p}^{1,2}\left((-4,3) \times \mathbb{R}^{d}\right)$ satisfying $\widetilde{\boldsymbol{w}}(-4, \cdot)=0$ and

$$
P \widetilde{\boldsymbol{w}}=\boldsymbol{f} I_{Q_{\kappa r}}
$$

where $\boldsymbol{f}:=P \boldsymbol{u}$. This is possible by Remark 5.2. In fact, $\widetilde{\boldsymbol{w}} \in W_{q}^{1,2}\left((-4,3) \times \mathbb{R}^{d}\right)$ for all $q \in(1, \infty)$ because $\boldsymbol{f} I_{Q_{\kappa r}} \in L_{q}\left((-4,3) \times \mathbb{R}^{d}\right)$ for all $q \in(1, \infty)$. Let

$$
\boldsymbol{w}(t, x)=\eta(t) \widetilde{\boldsymbol{w}}(t, x)
$$

where $\eta(t)$ is an infinitely differentiable function defined on $\mathbb{R}$ such that

$$
\eta(t)=1, \quad-2 \leq t \leq 1, \quad \eta(t)=0, \quad t \leq-3 \quad \text { or } \quad t \geq 2 .
$$

We see that $\boldsymbol{w} \in W_{p}^{1,2}\left(\mathbb{R}^{d+1}\right)$ and, in addition, $\boldsymbol{w} \in W_{q}^{1,2}\left(\mathbb{R}^{d+1}\right)$ for all $q \in(1, \infty)$. From Remark 5.2 (the estimate in Theorem 2.4 for $p=q$ ) we have

$$
\int_{Q_{\kappa r}}\left|D^{2} \boldsymbol{w}\right|^{p} d x d t \leq \int_{(-4,3) \times \mathbb{R}^{d}}\left|D^{2} \widetilde{\boldsymbol{w}}\right|^{p} d x d t \leq N \int_{Q_{\kappa r}} \mid \boldsymbol{f}^{p} d x d t
$$


where $N$ depends only on $d, m, p, \delta, K$, and the function $\omega$. Thus

$$
\begin{aligned}
\left(\left|D^{2} \boldsymbol{w}\right|^{p}\right)_{Q_{\kappa r}} & \leq N\left(|\boldsymbol{f}|^{p}\right)_{Q_{\kappa r}}, \\
\left(\left|D^{2} \boldsymbol{w}\right|^{p}\right)_{Q_{r}} & \leq N \kappa^{d+2}\left(|\boldsymbol{f}|^{p}\right)_{Q_{\kappa r}},
\end{aligned}
$$

where $N=N(d, m, p, \delta, K, \omega)$.

Now we set

$$
v=u-w .
$$

Then $\boldsymbol{v} \in W_{p}^{1,2}\left(\mathbb{R}^{d+1}\right), \boldsymbol{v} \in W_{q}^{1,2}\left(\mathbb{R}^{d+1}\right), q \in(1, \infty)$, and

$$
P \boldsymbol{v}=0 \text { in } Q_{\kappa r} .
$$

Define $\bar{P}$ by

$$
\bar{P} \boldsymbol{u}=-\boldsymbol{u}_{t}+\bar{A}^{\alpha \beta}(t) D_{\alpha \beta} \boldsymbol{u},
$$

where

$$
\bar{A}^{\alpha \beta}(t)=f_{B_{\kappa r / 2}} A^{\alpha \beta}(t, y) d y .
$$

Since $\boldsymbol{v} \in W_{p}^{1,2}\left(\mathbb{R}^{d+1}\right)$ and $\kappa / 2 \geq 4$, by Theorem 3.6 applied to the operator $\bar{P}$, we have

$$
f_{Q_{r}}\left|D^{2} \boldsymbol{v}(t, x)-\left(D^{2} \boldsymbol{v}\right)_{Q_{r}}\right|^{p} d x d t \leq N \kappa^{d+2}\left(|\bar{P} \boldsymbol{v}|^{p}\right)_{Q_{\kappa r / 2}}+N \kappa^{-p}\left(\left|D^{2} \boldsymbol{v}\right|^{p}\right)_{Q_{\kappa r / 2}} .
$$

Using the fact that $P \boldsymbol{v}=0$ in $Q_{\kappa r}$, we have

$$
\begin{aligned}
\left(|\bar{P} \boldsymbol{v}|^{p}\right)_{Q_{\kappa r / 2}} & =\left(|\bar{P} \boldsymbol{v}-P \boldsymbol{v}|^{p}\right)_{Q_{\kappa r / 2}} \\
& =f_{Q_{\kappa r / 2}}\left|\left(\bar{A}^{\alpha \beta}(t)-A^{\alpha \beta}(t, x)\right) D_{\alpha \beta} \boldsymbol{v}(t, x)\right|^{p} d x d t \\
& \leq N\left(f_{Q_{\kappa r / 2}}|\bar{A}(t)-A(t, x)|^{2 p} d x d t\right)^{1 / 2}\left(f_{Q_{\kappa r / 2}}\left|D^{2} \boldsymbol{v}\right|^{2 p} d x d t\right)^{1 / 2}
\end{aligned}
$$

where we see

$$
f_{Q_{\kappa r / 2}}|\bar{A}(t)-A(t, x)|^{2 p} d x d t \leq N f_{Q_{\kappa r / 2}}|\bar{A}(t)-A(t, x)| d x d t \leq N \omega(\kappa r / 2) .
$$

On the other hand, from Lemma 6.2 we see

$$
\left(f_{Q_{\kappa r / 2}}\left|D^{2} \boldsymbol{v}\right|^{2 p} d x d t\right)^{1 / 2} \leq N\left(f_{Q_{\kappa r}}\left|D^{2} \boldsymbol{v}\right|^{p} d x d t\right),
$$

where $N=N(d, m, p, \delta, K, \omega)$. Hence

$$
f_{Q_{r}}\left|D^{2} \boldsymbol{v}(t, x)-\left(D^{2} \boldsymbol{v}\right)_{Q_{r}}\right|^{p} d x d t \leq N\left(\kappa^{-p}+\kappa^{d+2} \omega(\kappa r)^{1 / 2}\right)\left(\left|D^{2} \boldsymbol{v}\right|^{p}\right)_{Q_{\kappa r}} .
$$


Note that

$$
\begin{aligned}
\left(\left|D^{2} \boldsymbol{v}\right|^{p}\right)_{Q_{\kappa r}} & \leq N\left(\left|D^{2} \boldsymbol{u}\right|^{p}\right)_{Q_{\kappa r}}+N\left(\left|D^{2} \boldsymbol{w}\right|^{p}\right)_{Q_{\kappa r}} \\
& \leq N\left(\left|D^{2} \boldsymbol{u}\right|^{p}\right)_{Q_{\kappa r}}+N\left(\mid \boldsymbol{f}^{p}\right)_{Q_{\kappa r}}
\end{aligned}
$$

where the second inequality is due to (12). Also note that, using the inequality (13), we have

$$
f_{Q_{r}}\left|D^{2} \boldsymbol{w}(t, x)-\left(D^{2} \boldsymbol{w}\right)_{Q_{r}}\right|^{p} d x d t \leq N\left(\left|D^{2} \boldsymbol{w}\right|^{p}\right)_{Q_{r}} \leq N \kappa^{d+2}\left(|\boldsymbol{f}|^{p}\right)_{Q_{\kappa r}} .
$$

Therefore,

$$
\begin{aligned}
& \quad f_{Q_{r}}\left|D^{2} \boldsymbol{u}(t, x)-\left(D^{2} \boldsymbol{u}\right)_{Q_{r}}\right|^{p} d x d t \\
& \quad \leq N f_{Q_{r}}\left|D^{2} \boldsymbol{v}(t, x)-\left(D^{2} \boldsymbol{v}\right)_{Q_{r}}\right|^{p} d x d t+N f_{Q_{r}}\left|D^{2} \boldsymbol{w}(t, x)-\left(D^{2} \boldsymbol{w}\right)_{Q_{r}}\right|^{p} d x d t \\
& \quad \leq N\left(\kappa^{-p}+\kappa^{d+2} \omega(\kappa r)^{1 / 2}\right)\left(\left|D^{2} \boldsymbol{u}\right|^{p}+|\boldsymbol{f}|^{p}\right)_{Q_{\kappa r}}+N \kappa^{d+2}\left(|\boldsymbol{f}|^{p}\right)_{Q_{\kappa r}} .
\end{aligned}
$$

The theorem is proved.

REMARK 6.4. As in Remark 3.8, one can replace the last term of (11) by

$$
N\left(\kappa^{-p}+\kappa^{d+2} \omega(\kappa r)^{1 / 2}\right)\left(\left|D^{2} \boldsymbol{u}\right|\right)_{Q_{\kappa r}}^{p} .
$$

If $g$ is a function defined on $\mathbb{R}$, we define $(g)_{(a, b)}$ to be

$$
(g)_{(a, b)}=f_{(a, b)} g(s) d s=(b-a)^{-1} \int_{a}^{b} g(s) d s .
$$

Especially, the maximal and sharp function of $g$ are defined by

$$
\begin{gathered}
M g(t)=\sup _{t \in(a, b)} f_{(a, b)}|g(s)| d s, \\
g^{\#}(t)=\sup _{t \in(a, b)} f_{(a, b)}\left|g(s)-(g)_{(a, b)}\right| d s,
\end{gathered}
$$

where the supremums are taken over all intervals $(a, b)$ containing $t$.

Using Theorem 6.3 and the argument in the proof of Corollary 3.2 in [23], we obtain the following corollary.

Corollary 6.5. Let $B^{\alpha}=C=0$. Then there exists a constant $N=$ $N(d, m, p, \delta, K, \omega)$ such that, for any $\boldsymbol{u} \in C_{c}^{\infty}\left(\mathbb{R}^{d+1}\right), \kappa \geq 8$, and $r \in(0,1 / \kappa]$, we have

$$
\begin{aligned}
f_{\left(-r^{2}, 0\right)} \mid \varphi(t) & -\left.(\varphi)_{\left(-r^{2}, 0\right)}\right|^{p} d t \\
& \leq N \kappa^{d+2}\left(\psi^{p}\right)_{\left(-(\kappa r)^{2}, 0\right)}+N\left(\kappa^{-p}+\kappa^{d+2} \omega(\kappa r)^{1 / 2}\right)\left(\varphi^{p}\right)_{\left(-(\kappa r)^{2}, 0\right)},
\end{aligned}
$$


where

$$
\varphi(t)=\left\|D^{2} \boldsymbol{u}(t, \cdot)\right\|_{L_{p}\left(\mathbb{R}^{d}\right)}, \quad \psi(t)=\|P \boldsymbol{u}(t, \cdot)\|_{L_{p}\left(\mathbb{R}^{d}\right)} .
$$

The result in the above corollary makes it possible to show that the sharp function of $\varphi(t)$ is pointwisely bounded by the $p$-th root of the maximal functions of $|\psi|^{p}$ and $|\varphi|^{p}$ if $\varphi(t)$ has compact support.

Lemma 6.6. Let $B^{\alpha}=C=0, R \in(0,1]$, and $\boldsymbol{u}$ be a function in $C_{c}^{\infty}\left(\mathbb{R}^{d+1}\right)$ such that $\boldsymbol{u}(t, x)=0$ for $t \notin\left(0, R^{4}\right)$. Then

$$
\begin{aligned}
& \varphi^{\#}\left(t_{0}\right) \leq N \kappa^{(d+2) / p}\left(M\left(\psi^{p}\right)\left(t_{0}\right)\right)^{1 / p} \\
&+N\left((\kappa R)^{2-2 / p}+\kappa^{-1}+\kappa^{(d+2) / p}(\omega(R))^{1 / 2 p}\right)\left(M\left(\varphi^{p}\right)\left(t_{0}\right)\right)^{1 / p}
\end{aligned}
$$

for all $\kappa \geq 8$ and $t_{0} \in \mathbb{R}$, where $N=N(d, m, p, \delta, K, \omega)$ and the functions $\varphi$ and $\psi$ are defined as in Corollary 6.5.

Proof. Fix $\kappa$ such that $\kappa \geq 8$. If $r \leq R / \kappa$, then $\kappa r \leq R \leq 1$ and $A_{\kappa r}^{\#} \leq A_{R}^{\#} \leq$ $\omega(R)$. Thus by Corollary 6.5,

$$
\begin{aligned}
& \left(\left|\varphi-(\varphi)_{\left(-r^{2}, 0\right)}\right|^{p}\right)_{\left(-r^{2}, 0\right)} \\
& \quad \leq N \kappa^{d+2}\left(\psi^{p}\right)_{\left(-(\kappa r)^{2}, 0\right)}+N\left(\kappa^{-p}+\kappa^{d+2}(\omega(R))^{1 / 2}\right)\left(\varphi^{p}\right)_{\left(-(\kappa r)^{2}, 0\right)} .
\end{aligned}
$$

By an appropriate translation of this inequality we have

$$
f_{(a, b)}\left|\varphi(t)-(\varphi)_{(a, b)}\right|^{p} d t \leq N \kappa^{d+2}\left(\psi^{p}\right)_{(c, b)}+N\left(\kappa^{-p}+\kappa^{d+2}(\omega(R))^{1 / 2}\right)\left(\varphi^{p}\right)_{(c, b)}
$$

if $(a, b)$ is an interval such that $b-a \leq R^{2} / \kappa^{2}$ and $c=b-\kappa^{2}(b-a)$. Note that, for $t_{0} \in(a, b)$,

$$
\left(\psi^{p}\right)_{(c, b)} \leq M\left(\psi^{p}\right)\left(t_{0}\right), \quad\left(\varphi^{p}\right)_{(c, b)} \leq M\left(\varphi^{p}\right)\left(t_{0}\right) .
$$

Thus by the Hölder's inequality it follows that

$$
\begin{aligned}
f_{(a, b)}\left|\varphi(t)-(\varphi)_{(a, b)}\right| \leq N \kappa^{(d+2) / p}\left(M\left(\psi^{p}\right)\left(t_{0}\right)\right)^{1 / p} & \\
& +N\left(\kappa^{-1}+\kappa^{(d+2) / p}(\omega(R))^{1 / 2 p}\right)\left(M\left(\varphi^{p}\right)\left(t_{0}\right)\right)^{1 / p},
\end{aligned}
$$

where $t_{0} \in(a, b)$ and $b-a \leq R^{2} / \kappa^{2}$. Now, if $(a, b)$ is an interval such that $t_{0} \in(a, b)$ and $b-a>R^{2} / \kappa^{2}$, then

$$
\begin{aligned}
f_{(a, b)}\left|\varphi(t)-(\varphi)_{(a, b)}\right| d t & \leq 2 f_{(a, b)} I_{\left(0, R^{4}\right)}(t)|\varphi(t)| d t \\
& \leq 2\left(f_{(a, b)} I_{\left(0, R^{4}\right)}(t) d t\right)^{1-1 / p}\left(f_{(a, b)}|\varphi(t)|^{p} d t\right)^{1 / p} \\
& \leq 2(\kappa R)^{2-2 / p}\left(M\left(\varphi^{p}\right)\left(t_{0}\right)\right)^{1 / p}
\end{aligned}
$$


Therefore, for all intervals $(a, b) \ni t_{0}$,

$$
\begin{aligned}
& f_{(a, b)}\left|\varphi(t)-(\varphi)_{(a, b)}\right| d t \leq N \kappa^{(d+2) / p}\left(M\left(\psi^{p}\right)\left(t_{0}\right)\right)^{1 / p} \\
& \quad+N\left((\kappa R)^{2-2 / p}+\kappa^{-1}+\kappa^{(d+2) / p}(\omega(R))^{1 / 2 p}\right)\left(M\left(\varphi^{p}\right)\left(t_{0}\right)\right)^{1 / p} .
\end{aligned}
$$

Taking the supremum of the left-hand side of the above inequality over all intervals $(a, b) \ni t_{0}$, we obtain the inequality in the lemma. The lemma is proved.

We use again the Hardy-Littlewood theorem and Fefferman-Stein theorem to derive the $L_{q, p^{-}}$estimate of second order derivatives of solutions to parabolic systems.

Corollary 6.7. Let $B^{\alpha}=C=0$ and $1<p<q<\infty$. Then there exists $R=R(d, m, p, \delta, K, \omega)$ such that, for any $\boldsymbol{u} \in C_{c}^{\infty}\left(\mathbb{R}^{d+1}\right)$ satisfying $\boldsymbol{u}(t, x)=0$ for $t \notin\left(0, R^{4}\right)$,

$$
\left\|D^{2} \boldsymbol{u}\right\|_{L_{q, p}\left(\mathbb{R}^{d+1}\right)} \leq N\|P \boldsymbol{u}\|_{L_{q, p}\left(\mathbb{R}^{d+1}\right)},
$$

where $N=N(d, m, p, q, \delta, K, \omega)$.

Proof. Let $\boldsymbol{u} \in C_{c}^{\infty}\left(\mathbb{R}^{d+1}\right)$ be such that $\boldsymbol{u}(t, x)=0$ for $t \notin\left(0, R^{4}\right), R \in(0,1]$, where $R$ will be specified below. Using the inequality in Lemma 6.6 as well as the Hardy-Littlewood theorem and the Fefferman-Stein theorem (recall that $q / p>1$ ), we arrive at

$$
\begin{aligned}
\left\|D^{2} \boldsymbol{u}\right\|_{L_{q, p}\left(\mathbb{R}^{d+1}\right)} \leq & N \kappa^{(d+2) / p}\|P \boldsymbol{u}\|_{L_{q, p}\left(\mathbb{R}^{d+1}\right)} \\
& +N\left((\kappa R)^{2-2 / p}+\kappa^{-1}+\kappa^{(d+2) / p}(\omega(R))^{1 / 2 p}\right)\left\|D^{2} \boldsymbol{u}\right\|_{L_{q, p}\left(\mathbb{R}^{d+1}\right)}
\end{aligned}
$$

for all $\kappa \geq 8$. Now we choose a large $\kappa$ and then a small $R$ such that

$$
N\left((\kappa R)^{2-2 / p}+\kappa^{-1}+\kappa^{(d+2) / p}(\omega(R))^{1 / 2 p}\right)<\frac{1}{2} .
$$

It then follows that

$$
\left\|D^{2} \boldsymbol{u}\right\|_{L_{q, p}\left(\mathbb{R}^{d+1}\right)} \leq N\|P \boldsymbol{u}\|_{L_{q, p}\left(\mathbb{R}^{d+1}\right)} .
$$

This finishes the proof.

Proof of Theorem 2.4. For the case $p=q$, the theorem is proved by Theorem 5.1 (also see Remark 5.2). For the case $q>p$, the theorem is proved by the same reasoning in the proof of Theorem 5.1, i.e., using Corollary 6.7 and the arguments in $[22]$.

7. Systems in divergence form with $\mathrm{VMO}_{x}$ coefficients in $L_{p}$. This section is devoted to proving the following theorem, which is a counterpart of Theorem 5.1 for systems in the divergence form.

Theorem 7.1. Let $\boldsymbol{u} \in \mathcal{H}_{p}^{1}\left(\mathbb{R}_{T}^{d+1}\right), T \in(-\infty, \infty]$. Then there exist constants $\lambda_{0} \geq 0$ and $N$, depending only on $d, m, p, \delta, K$, the function $\omega$, such that

$$
\lambda\|\boldsymbol{u}\|_{L_{p}\left(\mathbb{R}_{T}^{d+1}\right)}+\sqrt{\lambda}\|D \boldsymbol{u}\|_{L_{p}\left(\mathbb{R}_{T}^{d+1}\right)}+\left\|\boldsymbol{u}_{t}\right\|_{\mathbb{H}_{p}^{-1}\left(\mathbb{R}_{T}^{d+1}\right)} \leq N(\sqrt{\lambda}+1)\|\mathcal{P} \boldsymbol{u}-\lambda \boldsymbol{u}\|_{\mathbb{H}_{p}^{-1}\left(\mathbb{R}_{T}^{d+1}\right)}
$$


for all $\lambda \geq \lambda_{0}$. Moreover, for any $\lambda>\lambda_{0}$ and $\boldsymbol{f}, \boldsymbol{g}_{\alpha} \in L_{p}\left(\mathbb{R}_{T}^{d+1}\right)$, there exists a unique $\boldsymbol{u} \in \mathcal{H}_{p}^{1}\left(\mathbb{R}_{T}^{d+1}\right)$ satisfying

$$
\mathcal{P} \boldsymbol{u}-\lambda \boldsymbol{u}=\boldsymbol{f}+D_{\alpha} \boldsymbol{g}_{\alpha}
$$

Remark 7.2. As in Remark 5.2, Theorem 7.1 implies Theorem 2.5 for $p=q$.

For the proof of this theorem, we need a few auxiliary results. First, the following lemma is derived from Theorem 3.7 as Lemma 5.3 is derived from Theorem 3.6.

Lemma 7.3. Let $B^{\alpha}=\hat{B}^{\alpha}=0, C=0, \mu, \nu \in(1, \infty), 1 / \mu+1 / \nu=1$, and $R \in(0, \infty)$. Assume $\boldsymbol{u} \in C_{c}^{\infty}\left(\mathbb{R}^{d+1}\right)$ vanishing outside $Q_{R}$ and $\mathcal{P} \boldsymbol{u}=D_{\alpha} \boldsymbol{g}_{\alpha}$, where $\boldsymbol{g}=\left(\boldsymbol{g}_{\alpha}\right) \in L_{p}\left(\mathbb{R}^{d+1}\right)$. Then there exists a constant $N=N(d, m, q, \delta, K, \mu)$ such that

$$
\begin{aligned}
\left(\left|D \boldsymbol{u}-(D \boldsymbol{u})_{Q_{r}\left(t_{0}, x_{0}\right)}\right|^{q}\right)_{Q_{r}\left(t_{0}, x_{0}\right)} & \leq N \kappa^{-q}\left(|D \boldsymbol{u}|^{q}\right)_{Q_{\kappa r}\left(t_{0}, x_{0}\right)} \\
& +N \kappa^{d+2}\left(\left(|\boldsymbol{g}|^{q}\right)_{Q_{\kappa r}\left(t_{0}, x_{0}\right)}+\omega(R)^{1 / \nu}\left(|D \boldsymbol{u}|^{q \mu}\right)_{Q_{\kappa r}\left(t_{0}, x_{0}\right)}^{1 / \mu}\right)
\end{aligned}
$$

for any $r \in(0, \infty), \kappa \geq 4$, and $\left(t_{0}, x_{0}\right) \in \mathbb{R}^{d+1}$.

Here is a counterpart of Proposition 5.4 which is proved by the same method.

Proposition 7.4. Let $B^{\alpha}=\hat{B}^{\alpha}=0$ and $C=0$. Then there exist constants $R=$ $R(d, m, p, \delta, K, \omega)$ and $N=N(d, m, p, \delta, K)$ such that, for $\boldsymbol{u} \in C_{c}^{\infty}\left(\mathbb{R}^{d+1}\right)$ vanishing outside $Q_{R}$ and $\boldsymbol{g}=\left(\boldsymbol{g}_{\alpha}\right) \in L_{p}\left(\mathbb{R}^{d+1}\right)$ satisfying $\mathcal{P} \boldsymbol{u}=D_{\alpha} \boldsymbol{g}_{\alpha}$, we have

$$
\|D \boldsymbol{u}\|_{L_{p}\left(\mathbb{R}^{d+1}\right)} \leq N\|\boldsymbol{g}\|_{L_{p}\left(\mathbb{R}^{d+1}\right)} .
$$

Corollary 7.5. Let $\boldsymbol{f}, \boldsymbol{g}=\left(\boldsymbol{g}_{\alpha}\right) \in L_{p}\left(\mathbb{R}^{d+1}\right)$ and $\boldsymbol{u} \in C_{c}^{\infty}\left(\mathbb{R}^{d+1}\right)$. Then there exist $R, \lambda_{0}$, and $N$, depending only on $d, m, p, \delta, K$, and the function $\omega$, such that if $\boldsymbol{u}$ vanishes outside $Q_{R}$ then we have

$$
\sqrt{\lambda}\|D \boldsymbol{u}\|_{L_{p}\left(\mathbb{R}^{d+1}\right)}+\lambda\|\boldsymbol{u}\|_{L_{p}\left(\mathbb{R}^{d+1}\right)} \leq N \sqrt{\lambda}\|\boldsymbol{g}\|_{L_{p}\left(\mathbb{R}^{d+1}\right)}+N\|\boldsymbol{f}\|_{L_{p}\left(\mathbb{R}^{d+1}\right)}
$$

provided that $\lambda \geq \lambda_{0}$ and

$$
\mathcal{P} \boldsymbol{u}-\lambda \boldsymbol{u}=D_{\alpha} \boldsymbol{g}_{\alpha}+\boldsymbol{f}
$$

Proof. Due to Proposition 7.4, the corollary can be proved by using the aforementioned idea of Agmon. See the proof of Lemma 5.5 of [22].

Theorem 7.6. Let $\boldsymbol{u} \in C_{c}^{\infty}\left(\mathbb{R}^{d+1}\right)$. There exist $\lambda_{0}$ and $N$ depending only on $d, m, p, \delta, K$, and the function $\omega$ such that

$$
\begin{aligned}
&\left\|\boldsymbol{u}_{t}\right\|_{\mathbb{H}_{p}^{-1}\left(\mathbb{R}^{d+1}\right)}+\sqrt{\lambda}\|D \boldsymbol{u}\|_{L_{p}\left(\mathbb{R}^{d+1}\right)}+\lambda\|\boldsymbol{u}\|_{L_{p}\left(\mathbb{R}^{d+1}\right)} \\
& \leq N \sqrt{\lambda}\|\boldsymbol{g}\|_{L_{p}\left(\mathbb{R}^{d+1}\right)}+N\|\boldsymbol{f}\|_{L_{p}\left(\mathbb{R}^{d+1}\right)}
\end{aligned}
$$

provided that $\lambda \geq \lambda_{0}$ and

$$
\mathcal{P} \boldsymbol{u}-\lambda \boldsymbol{u}=D_{\alpha} \boldsymbol{g}_{\alpha}+\boldsymbol{f}
$$


Moreover, there exists $N$ depending only on $d, m, p, \delta, K$, and $\omega$ such that

$$
\begin{aligned}
\left\|\boldsymbol{u}_{t}\right\|_{\mathbb{H}_{p}^{-1}\left(\mathbb{R}^{d+1}\right)}+\sqrt{\lambda}\|D \boldsymbol{u}\|_{L_{p}\left(\mathbb{R}^{d+1}\right)}+\lambda\|\boldsymbol{u}\|_{L_{p}\left(\mathbb{R}^{d+1}\right)} & \\
& \leq N(\sqrt{\lambda}+1)\|\mathcal{P} \boldsymbol{u}-\lambda \boldsymbol{u}\|_{\mathbb{H}_{p}^{-1}\left(\mathbb{R}^{d+1}\right)}
\end{aligned}
$$

provided that $\lambda \geq \lambda_{0}$.

Proof. The first part of the theorem is obtained by noting that $(1-\Delta)^{-1 / 2}$ and $(1-\Delta)^{-1 / 2} D$ are bounded operators on $L_{p}\left(\mathbb{R}^{d+1}\right)$ and using the standard partition of unity technique. Next we prove the second part. Denote

$$
\boldsymbol{h}=\mathcal{P} \boldsymbol{u}-\lambda \boldsymbol{u}, \quad \overline{\boldsymbol{f}}=(1-\Delta)^{-1} \boldsymbol{h}, \quad \overline{\boldsymbol{g}}_{\alpha}=-D_{\alpha} \overline{\boldsymbol{f}} .
$$

Note that we have

$$
D_{\alpha} \overline{\boldsymbol{g}}_{\alpha}+\overline{\boldsymbol{f}}=(1-\Delta) \overline{\boldsymbol{f}}=\boldsymbol{h} .
$$

Again due to the boundedness of $(1-\Delta)^{-1 / 2}$ and $(1-\Delta)^{-1 / 2} D_{\alpha}$ in $L_{p}\left(\mathbb{R}^{d+1}\right)$, we have $\boldsymbol{h} \in \mathbb{H}_{p}^{-1}\left(\mathbb{R}^{d+1}\right), \overline{\boldsymbol{f}} \in L_{p}\left(\mathbb{R}^{d+1}\right), \overline{\boldsymbol{g}}=\left(\overline{\boldsymbol{g}}_{\alpha}\right) \in L_{p}\left(\mathbb{R}^{d+1}\right)$ and

$$
\|\overline{\boldsymbol{g}}\|_{L_{p}\left(\mathbb{R}^{d+1}\right)}+\|\overline{\boldsymbol{f}}\|_{L_{p}\left(\mathbb{R}^{d+1}\right)} \leq N\|\boldsymbol{h}\|_{\mathbb{H}_{p}^{-1}\left(\mathbb{R}^{d+1}\right)} .
$$

By the first part with $\overline{\boldsymbol{f}}$ and $\overline{\boldsymbol{g}}_{\alpha}$ in place of $\boldsymbol{f}$ and $\boldsymbol{g}_{\alpha}$, we bound the left-hand side of (14) by (recall $\boldsymbol{h}=D_{\alpha} \overline{\boldsymbol{g}}_{\alpha}+\overline{\boldsymbol{f}}$ )

$$
N \sqrt{\lambda}\|\overline{\boldsymbol{g}}\|_{L_{p}\left(\mathbb{R}^{d+1}\right)}+N\|\overline{\boldsymbol{f}}\|_{L_{p}\left(\mathbb{R}^{d+1}\right)} \leq N(\sqrt{\lambda}+1)\|\boldsymbol{h}\|_{\mathbb{H}_{p}^{-1}\left(\mathbb{R}^{d+1}\right)} .
$$

The theorem is proved.

Proof of Theorem 7.1 As usual we only have to prove the apriori estimate. For $T=\infty$, this is given by Theorem 7.6. For $T<\infty$, we again make use of the argument in Theorem 2.1 in [23]. The theorem is proved.

8. Proof of Theorem 2.5. Throughout this section, let $\mathcal{P}$ be the operator defined in (2), where the matrices $A^{\alpha \beta}, B^{\alpha}, \hat{B}^{\alpha}$ and $C$ satisfy Assumption 2.1 and 2.3. We shall use the following Sobolev embedding type estimate.

Lemma 8.1. Let $T \in(-\infty, \infty], r \in(0, \infty), 1<q \leq p<\infty$, and assume that

$$
1 / q-1 / p \leq 1 /(d+2) .
$$

Then for any function $\boldsymbol{u} \in \mathcal{H}_{q}^{1}\left(\mathbb{R}_{T}^{d+1}\right)$ we have $\boldsymbol{u} \in L_{p}\left(\mathbb{R}_{T}^{d+1}\right)$ and

$$
\|\boldsymbol{u}\|_{L_{p}\left(\mathbb{R}_{T}^{d+1}\right)} \leq N\|\boldsymbol{u}\|_{\mathcal{H}_{q}^{1}\left(\mathbb{R}_{T}^{d+1}\right)},
$$

where $N>0$ depends only on $d, m, q$ and $p$.

Proof. This result is implied by Theorem 7.1. See the proof of, for example, Lemma 8.1 of [23].

With the aid of Lemma 8.1, we can get a counterpart of Lemma 6.2.

Lemma 8.2. Let $r \in(0,1], q>1$, and $B^{\alpha}=\hat{B}^{\alpha}=C=0$. Assume that $\boldsymbol{u} \in \mathcal{H}_{q, l o c}^{1}\left(\mathbb{R}^{d+1}\right)$ and $\mathcal{P} \boldsymbol{u}=0$ in $Q_{2 r}$, then $D \boldsymbol{u} \in L_{p}\left(Q_{r}\right)$ and

$$
\left(|D \boldsymbol{u}|^{p}\right)_{Q_{r}}^{1 / p} \leq N\left(|D \boldsymbol{u}|^{q}\right)_{Q_{2 r}}^{1 / q}
$$


where $N$ depends only on $d, m, p, q, K, \delta$ and $\omega$.

Here come the key estimates in proving Theorem 2.5. The first one is an analogue of Theorem 6.3, which is proved in a similar way. The second is a counterpart of Corollary 6.5.

TheOrem 8.3. Let $B^{\alpha}=\hat{B}^{\alpha}=0, C=0, \boldsymbol{g}=\left(\boldsymbol{g}_{\alpha}\right) \in L_{p, l o c}\left(\mathbb{R}^{d+1}\right), \boldsymbol{u} \in$ $\mathcal{H}_{p, l o c}^{1}\left(\mathbb{R}^{d+1}\right), \kappa \geq 8$ and $r \in(0,1 / \kappa]$. Suppose $\mathcal{P} \boldsymbol{u}=D_{\alpha} \boldsymbol{g}_{\alpha}$. Then there exists a constant $N$ depending only on $d, m, p, \delta, K, \omega$ such that

$$
\begin{aligned}
f_{Q_{r}}\left|D \boldsymbol{u}-(D \boldsymbol{u})_{Q_{r}}\right|^{p} d x d t \\
\quad \leq N \kappa^{d+2}\left(|\boldsymbol{g}|^{p}\right)_{Q_{\kappa r}}+N\left(\kappa^{-p}+\kappa^{d+2} \omega(\kappa r)^{1 / 2}\right)\left(|D \boldsymbol{u}|^{p}\right)_{Q_{\kappa r}} .
\end{aligned}
$$

Proof. Without loss of generality we can assume that $\boldsymbol{u} \in \mathcal{H}_{p}^{1}\left(\mathbb{R}^{d+1}\right)$. Due to Remark 7.2 there exists $\boldsymbol{v} \in \mathcal{H}_{p}^{1}\left((-4,0) \times \mathbb{R}^{d}\right)$ satisfying

$$
\mathcal{P} \boldsymbol{v}=D_{\alpha}\left(I_{Q_{\kappa r}} \boldsymbol{g}_{\alpha}\right) .
$$

Note that $\kappa r \leq 1$ so that $\boldsymbol{v}(t, x)=0$ for $t \leq-1$. Remark 7.2 also gives that

$$
\int_{(-4,0) \times \mathbb{R}^{d}}|D \boldsymbol{v}|^{p} d x d t \leq N \int_{Q_{\kappa r}}|\boldsymbol{g}|^{p} d x d t
$$

Now we set $\boldsymbol{w}=\boldsymbol{u}-\boldsymbol{v}$ and notice that $\boldsymbol{w} \in \mathcal{H}_{p}^{1}\left((-4,0) \times \mathbb{R}^{d}\right)$ and $\boldsymbol{w}$ satisfies $\mathcal{P} \boldsymbol{w}=0$ in $Q_{\kappa r}$. Define $\bar{P}$ by

$$
\bar{P} \boldsymbol{u}=-\boldsymbol{u}_{t}+\bar{A}^{\alpha \beta}(t) D_{\alpha \beta} \boldsymbol{u},
$$

where

$$
\bar{A}^{\alpha \beta}(t)=f_{B_{\kappa r / 2}} A^{\alpha \beta}(t, y) d y .
$$

Then it is clear that in $Q_{\kappa r}$, we have

$$
\bar{P} \boldsymbol{w}=D_{\alpha} \overline{\boldsymbol{g}}_{\alpha}, \quad \overline{\boldsymbol{g}}_{\alpha}=\left(\bar{A}^{\alpha \beta}-A^{\alpha \beta}\right) D_{\beta} \boldsymbol{w} .
$$

Applying Theorem 3.7 to $\boldsymbol{w}$ instead of $\boldsymbol{u}$, using Hölder's inequality and Lemma 8.2, we obtain

$$
\begin{gathered}
f_{Q_{r}}\left|D \boldsymbol{w}(t, x)-(D \boldsymbol{w})_{Q_{r}}\right|^{p} d x d t \leq N \kappa^{d+2}\left(|\overline{\boldsymbol{g}}|^{p}\right)_{Q_{\kappa r / 2}}+N \kappa^{-p}\left(|D \boldsymbol{w}|^{p}\right)_{Q_{\kappa r / 2}} \\
\leq N \kappa^{d+2}\left(|A-\bar{A}|^{2 p}\right)_{Q_{\kappa r / 2}}^{1 / 2}\left(|D \boldsymbol{w}|^{2 p}\right)_{Q_{\kappa r / 2}}^{1 / 2}+N \kappa^{-p}\left(|D \boldsymbol{w}|^{p}\right)_{Q_{\kappa r / 2}} \\
\leq N \kappa^{d+2} \omega(\kappa r)^{1 / 2}\left(|D \boldsymbol{w}|^{p}\right)_{Q_{\kappa r}}+N \kappa^{-p}\left(|D \boldsymbol{w}|^{p}\right)_{Q_{\kappa r / 2}} .
\end{gathered}
$$

Observe that

$$
\left(|D \boldsymbol{w}|^{p}\right)_{Q_{\kappa r / 2}} \leq N\left(|D \boldsymbol{w}|^{p}\right)_{Q_{\kappa r}} \leq N\left(|D \boldsymbol{u}|^{p}\right)_{Q_{\kappa r}}+N\left(|D \boldsymbol{v}|^{p}\right)_{Q_{\kappa r}} .
$$


These together with (16) imply that the left-hand side of (15) is bounded by

$$
\begin{aligned}
N f_{Q_{r}}\left|D \boldsymbol{w}-(D \boldsymbol{w})_{Q_{r}}\right|^{p} d x d t+N f_{Q_{r}}|D \boldsymbol{v}|^{p} d x d t \\
\quad \leq N\left(\kappa^{-p}+\kappa^{d+2} \omega(\kappa r)^{1 / 2}\right)\left(|D \boldsymbol{u}|^{p}\right)_{Q_{\kappa r}}+N \kappa^{d+2}\left(|\boldsymbol{g}|^{p}\right)_{Q_{\kappa r}} .
\end{aligned}
$$

The theorem is proved.

Remark 8.4. As in Remark 3.8, one can replace the last term of (15) by

$$
N\left(\kappa^{-p}+\kappa^{d+2} \omega(\kappa r)^{1 / 2}\right)(|D \boldsymbol{u}|)_{Q_{\kappa r}}^{p} .
$$

Recall the notation $(g)_{(a, b)}, M g(t)$ and $g^{\#}(t)$ defined in Section 6 .

Corollary 8.5. Let $B^{\alpha}=\hat{B}^{\alpha}=0, C=0$. Assume $\boldsymbol{u} \in \mathcal{H}_{p}^{1}\left((S, T) \times \mathbb{R}^{d}\right)$, $\boldsymbol{g}=\left(\boldsymbol{g}_{\alpha}\right) \in L_{p}\left((S, T) \times \mathbb{R}^{d}\right)$ for some $-\infty<S<T<\infty$ and

$$
\mathcal{P} \boldsymbol{u}=D_{\alpha} \boldsymbol{g}_{\alpha} \quad \text { in } \quad(S, T) \times \mathbb{R}^{d} .
$$

Then there exists a constant $N=N(d, m, p, \delta, K, \omega)$ such that, for any $\kappa \geq 8$ and $r \in(0,1 / \kappa]$, we have

$$
\begin{aligned}
f_{\left(-r^{2}, 0\right)} \mid \varphi(t)- & \left.(\varphi)_{\left(-r^{2}, 0\right)}\right|^{p} d t \\
& \leq N \kappa^{d+2}\left(\psi^{p}\right)_{\left(-(\kappa r)^{2}, 0\right)}+N\left(\kappa^{-p}+\kappa^{d+2} \omega(\kappa r)^{1 / 2}\right)\left(\varphi^{p}\right)_{\left(-(\kappa r)^{2}, 0\right)},
\end{aligned}
$$

where

$$
\varphi(t)=\|D \boldsymbol{u}(t, \cdot)\|_{L_{p}\left(\mathbb{R}^{d}\right)}, \quad \psi(t)=\|\boldsymbol{g}(t, \cdot)\|_{L_{p}\left(\mathbb{R}^{d}\right)} .
$$

In the same fashion, we derive a counterpart of Lemma 6.6.

Lemma 8.6. Let $B^{\alpha}=\hat{B}^{\alpha}=0, C=0, R \in(0,1]$, and $\boldsymbol{u} \in C_{c}^{\infty}\left(\mathbb{R}^{d+1}\right), \boldsymbol{g}=$ $\left(\boldsymbol{g}_{\alpha}\right) \in L_{p}\left(\mathbb{R}^{d+1}\right)$ such that $\boldsymbol{u}(t, x)=0$ for $t \notin\left(0, R^{4}\right)$. Assume $\mathcal{P} \boldsymbol{u}=D_{\alpha} \boldsymbol{g}_{\alpha}$. Then

$$
\begin{aligned}
\varphi^{\#}\left(t_{0}\right) \leq N \kappa^{(d+2) / p} M & \left(\psi^{p}\right)\left(t_{0}\right)^{1 / p} \\
& +N\left((\kappa R)^{2-2 / p}+\kappa^{-1}+\kappa^{(d+2) / p} \omega(R)^{1 / 2 p}\right) M\left(\varphi^{p}\right)\left(t_{0}\right)^{1 / p}
\end{aligned}
$$

for all $\kappa \geq 8$ and $t_{0} \in \mathbb{R}$, where $N=N(d, m, p, \delta, K, \omega)$ and the functions $\varphi, \psi$ are defined as in Corollary 8.5.

Corollary 8.7. Let $B^{\alpha}=\hat{B}^{\alpha}=0, C=0,1<p<q<\infty$ and $\boldsymbol{u} \in C_{c}^{\infty}\left(\mathbb{R}^{d+1}\right), \boldsymbol{g}=\left(\boldsymbol{g}_{\alpha}\right) \in L_{q, p}\left(\mathbb{R}^{d+1}\right)$. Assume $\mathcal{P} \boldsymbol{u}=D_{\alpha} \boldsymbol{g}_{\alpha}$. Then there exists $R=R(d, m, p, \delta, K, \omega)$ such that, under the assumption $\boldsymbol{u}(t, x)=0$ for $t \notin\left(0, R^{4}\right)$,

$$
\|D \boldsymbol{u}\|_{L_{q, p}\left(\mathbb{R}^{d+1}\right)} \leq N\|\boldsymbol{g}\|_{L_{q, p}\left(\mathbb{R}^{d+1}\right)},
$$

where $N=N(d, m, p, q, \delta, K, \omega)$. 
Proof. It follows from Lemma 8.6, the Hardy-Littlewood theorem, and the Fefferman-Stein theorem.

By using a partition of unity, we further conclude the following corollary.

Corollary 8.8. Let $B^{\alpha}=\hat{B}^{\alpha}=0, C=0,1<p<q<\infty$ and $\boldsymbol{u} \in C_{c}^{\infty}\left(\mathbb{R}^{d+1}\right), \boldsymbol{g}=\left(\boldsymbol{g}_{\alpha}\right) \in L_{q, p}\left(\mathbb{R}^{d+1}\right)$. Assume $\mathcal{P} \boldsymbol{u}=D_{\alpha} \boldsymbol{g}_{\alpha}$. Then we have

$$
\|D \boldsymbol{u}\|_{L_{q, p}\left(\mathbb{R}^{d+1}\right)} \leq N\|\boldsymbol{g}\|_{L_{q, p}\left(\mathbb{R}^{d+1}\right)}+N\|\boldsymbol{u}\|_{L_{q, p}\left(\mathbb{R}^{d+1}\right)}
$$

where $N=N(d, m, p, q, \delta, K, \omega)$.

Proof of Theorem 2.5. As usual, it suffices to derive the apriori estimate. For the case $p=q$, the theorem follows from Theorem 7.1 and Remark 7.2. For the case $q>p$, the theorem is proved by again the same reasoning as in the proof of Theorem 7.1, i.e., using Corollary 8.8, Agmon's idea, and the arguments in [22]. Finally, in the case $q<p$, we use duality argument.

9. Proof of Theorem 2.6 and 2.7. In this section, we prove Theorem 2.6 and 2.7. Roughly speaking, the idea is that solutions of elliptic systems can be considered as steady state solutions of the corresponding parabolic systems. Therefore, the estimates of parabolic systems which we derived imply the estimates of elliptic systems.

Proof of Theorem 2.6. As usual, it suffices to prove (3). Let $\eta$ be a smooth function on $\mathbb{R}$ supported on $[-2,2]$ and $\eta(t)=1$ on $[-1,1]$. For a fixed $T>0$, denote $\boldsymbol{v}(t, x)=\eta(t / T) \boldsymbol{u}(x)$. Then it is clear that $\boldsymbol{v} \in W_{p}^{1,2}\left(\mathbb{R}^{d+1}\right)$, and

$$
(P-\lambda) \boldsymbol{v}(t, x)=-T^{-1} \eta^{\prime}(t / T) \boldsymbol{u}(x)+\eta(t / T)(L-\lambda) \boldsymbol{u}(x) .
$$

Thanks to Theorem 5.1, we have

$$
\lambda\|\boldsymbol{v}\|_{L_{p}\left(\mathbb{R}^{d+1}\right)}+\sqrt{\lambda}\|D \boldsymbol{v}\|_{L_{p}\left(\mathbb{R}^{d+1}\right)}+\left\|D^{2} \boldsymbol{v}\right\|_{L_{p}\left(\mathbb{R}^{d+1}\right)} \leq N\|P \boldsymbol{v}-\lambda \boldsymbol{v}\|_{L_{p}\left(\mathbb{R}^{d+1}\right)} .
$$

This combined with (17) and the triangle inequality gives

$$
\begin{aligned}
& \|\eta(t / T)\|_{L_{p}(\mathbb{R})}\left(\lambda\|\boldsymbol{u}\|_{L_{p}\left(\mathbb{R}^{d}\right)}+\sqrt{\lambda}\|D \boldsymbol{u}\|_{L_{p}\left(\mathbb{R}^{d}\right)}+\left\|D^{2} \boldsymbol{u}\right\|_{L_{p}\left(\mathbb{R}^{d}\right)}\right) \\
& \quad \leq N\|\eta(t / T)\|_{L_{p}(\mathbb{R})}\|L \boldsymbol{u}-\lambda \boldsymbol{u}\|_{L_{p}\left(\mathbb{R}^{d}\right)}+N T^{-1}\left\|\eta^{\prime}(t / T)\right\|_{L_{p}\left(\mathbb{R}_{T}\right)}\|\boldsymbol{u}\|_{L_{p}\left(\mathbb{R}^{d}\right)} .
\end{aligned}
$$

Therefore,

$$
\begin{aligned}
T^{1 / p}\|\eta\|_{L_{p}(\mathbb{R})} & \left(\lambda\|\boldsymbol{u}\|_{L_{p}\left(\mathbb{R}^{d}\right)}+\sqrt{\lambda}\|D \boldsymbol{u}\|_{L_{p}\left(\mathbb{R}^{d}\right)}+\left\|D^{2} \boldsymbol{u}\right\|_{L_{p}\left(\mathbb{R}^{d}\right)}\right) \\
& \leq N T^{1 / p}\|\eta\|_{L_{p}(\mathbb{R})}\|L \boldsymbol{u}-\lambda \boldsymbol{u}\|_{L_{p}\left(\mathbb{R}^{d}\right)}+N T^{-1+1 / p}\left\|\eta^{\prime}\right\|_{L_{p}(\mathbb{R})}\|\boldsymbol{u}\|_{L_{p}\left(\mathbb{R}^{d}\right)} .
\end{aligned}
$$

Letting $T \rightarrow \infty$ yields (3). The theorem is proved.

Proof of Theorem 2.7. It is derived from Theorem 7.1 in the same way as Theorem 2.6 is derived from Theorem 5.1. We omit the details.

Acknowledgement. The authors are grateful to Nicolai V. Krylov for very helpful discussions on the subject. They also thank Seick Kim and the referees for many useful comments on earlier versions of the manuscript. 


\section{REFERENCES}

[1] E. Acerbi and G. Mingione, Gradient estimates for a class of parabolic systems, Duke Math. J., 136:2 (2007), pp. 285-320.

[2] P. Acquistapace, On BMO regularity for linear elliptic systems, Ann. Mat. Pura Appl., (4) 161 (1992), pp. 231-269.

[3] P. Auscher, On necessary and sufficient conditions for $L^{p}$ estimates of Riesz transforms associated to elliptic operators on $R^{n}$ and related estimates, Mem. Amer. Math. Soc., 186:871 (2007), xviii+75 pp.

[4] S. Byun, $W^{1, p}$ regularity theory for parabolic equations in divergence form, J. Evol. Equ., 7:3 (2007), pp. 415-428.

[5] S. Byun, H. Chen, M. Kim And L. Wang, L ${ }^{p}$ regularity theory for linear elliptic systems, Discrete Contin. Dyn. Syst., 18:1 (2007), pp. 121-134

[6] S. Campanato, Sistemi ellittici in forma divergenza. Regolaritá all'interno. (Italian) [Elliptic systems in divergence form. Interior regularity], Quaderni. [Publications] Scuola Normale Superiore Pisa, Pisa, 1980. 187 pp.

[7] F. Chiarenza, M. Frasca And P. Longo, Interior $W^{2, p}$ estimates for nondivergence elliptic equations with discontinuous coefficients, Ricerche Mat., 40 (1991), pp. 149-168.

[8] F. Chiarenza, M. Frasca and P. Longo, $W^{2, p}$-solvability of the Dirichlet problem for nondivergence elliptic equations with VMO coefficients, Trans. Amer. Math. Soc., 336 (1993), pp. 841-853.

[9] F. Chiarenza, M. Franciosi, M. Frasca, $L^{p}$-estimates for linear elliptic systems with discontinuous coefficients, Atti Accad. Naz. Lincei Cl. Sci. Fis. Mat. Natur. Rend. Lincei (9) Mat. Appl., 5:1 (1994), pp. 27-32.

[10] R. Denk, M. Hieber And J. Prüss, R-boundedness, Fourier multipliers and problems of elliptic and parabolic type, Mem. Amer. Math. Soc., 166 (2003).

[11] G. Di FAZIO, $L^{p}$ estimates for divergence form elliptic equations with discontinuous coefficients, Boll. Un. Mat. Ital. A, (7) 10:2 (1996), pp. 409-420.

[12] G. Di Fazio And M. FAnciullo, Gradient estimates for elliptic systems in CarnotCarathéodory spaces, Comment. Math. Univ. Carolin., 43:4 (2002), pp. 605-618.

[13] H. Dong AND D. KIM, Elliptic equations in divergence form with partially BMO coefficients, Arch. Ration. Mech. Anal., 196:1 (2010), pp. 25-70.

[14] H. Dong AND D, KIm, On the $L_{p}$-solvability of higher order parabolic and elliptic systems with $B M O$ coefficients, submitted (2009).

[15] A. Friedman, Partial Differential Equations of Parabolic Type, (1964) Prentice-Hall, Englewood Cliffs, N.J.

[16] M. Giaquinta, Introduction to regularity theory for nonlinear elliptic systems, Lectures in Mathematics ETH Zürich. Birkhäuser Verlag, Basel, 1993.

[17] R. Haller-Dintelmann, H. Heck and M. Hieber, $L^{p}-L^{q}$-estimates for parabolic systems in non-divergence form with VMO coefficients, J. London Math. Soc., (2) 74:3 (2006), pp. $717-736$.

[18] D. KIM, Parabolic equations with measurable coefficients, II, J. Math. Anal. Appl., 334:1 (2007), pp. 534-548.

[19] D. KIm AND N. V. KRYLOV, Elliptic differential equations with coefficients measurable with respect to one variable and VMO with respect to the others, SIAM J. Math. Anal., 39:2 (2007), pp. 489-506.

[20] D. Kim and N. V. Krylov, Parabolic equations with measurable coefficients, Potential Anal., 26:4 (2007), pp. 345-361.

[21] N. V. KRYlov, Parabolic equations in $L_{p}$-spaces with mixed norms, Algebra i Analiz, 14:4 (2002), pp. 91-106 (in Russian); English translation: St. Petersburg Math. J., 14:4 (2003), pp. 603-614.

[22] N. V. Krylov, Parabolic and elliptic equations with VMO coefficients, Comm. Partial Differential Equations, 32:3 (2007), pp. 453-475.

[23] N. V. Krylov, Parabolic equations with VMO coefficients in spaces with mixed norms, J. Funct. Anal., 250 (2007), pp. 521-558.

[24] S. LeOnardi, J. Kottas AND J. Stara, Hölder regularity of the solutions of some classes of elliptic systems in convex nonsmooth domains, Nonlinear Anal., 60 (2005), pp. 925-944.

[25] G. Lieberman, A mostly elementary proof of Morrey space estimates for elliptic and parabolic equations with VMO coefficients, J. Funct. Anal., 201:2 (2003), pp. 457-479.

[26] D. Palagachev and L. Softova, A priori estimates and precise regularity for parabolic systems with discontinuous data, Discrete Contin. Dyn. Syst., 13:3 (2005), pp. 721-742.

[27] D. Palagachev and L. Softova, Characterization of the interior regularity for parabolic 
systems with discontinuous coefficients, Atti. Accad. Naz. Lincei Cl. Sci. Fis. Mat. Natur. Rend. Lincei, (9) Mat. Appl., 16 (2005), pp. 125-132.

[28] D. Palagachev and L. Softova, Fine regularity for elliptic systems with discontinuous ingredients, Arch. Math. (Basel), 86:2 (2006), pp. 145-153.

[29] M. A. RAgusa, Local Hölder regularity for solutions of elliptic systems, Duke Math. J., 113 (2002), pp. 385-397.

[30] V. A. SolonNikov, On boundary value problems for linear parabolic systems of differential equations of general form, (Russian) Trudy Mat. Inst. Steklov., 83 (1965), pp. 3-163.

[31] B. Stroffolini, Elliptic systems of PDE with BMO-coefficients, Potential Anal., 15 (2001), pp. 285-299. 